\title{
Radiative rates for E1, E2, M1, and M2 transitions in F-like ions with $55 \leq \mathrm{Z} \leq 73$
}

\author{
Kanti M. Aggarwal ${ }^{\mathrm{a}, \text {,* }}$ \\ ${ }^{a}$ Astrophysics Research Centre, School of Mathematics and Physics, Queen's University Belfast, \\ Belfast BT7 1NN, Northern Ireland, UK
}

\begin{abstract}
Energy levels, radiative rates and lifetimes are reported for $19 \mathrm{~F}$-like ions with $55 \leq \mathrm{Z} \leq 73$, among 113 levels of the $2 \mathrm{~s}^{2} 2 \mathrm{p}^{5}, 2 \mathrm{~s} 2 \mathrm{p}^{6}, 2 \mathrm{~s}^{2} 2 \mathrm{p}^{4} 3 \ell, 2 \mathrm{~s} 2 \mathrm{p}^{5} 3 \ell$, and $2 \mathrm{p}^{6} 3 \ell$ configurations. The general-purpose relativistic atomic structure package (GRASP) has been adopted for the calculations, and radiative rates (and other associated parameters, such as oscillator 'strengths and line strengths) are listed for all E1, E2, M1, and M2 transitions of the ions. Comparisons are made with 'earlier available theoretical and experimental energies, especially for Ba XLVIII. Nevertheless, calculations have also been performed with the flexible atomic code (FAC), and with a much larger configuration interaction with up to 38089 levels, for further accuracy assessments, particularly for energy levels.
\end{abstract}

Received: 9 November 2017, Accepted: 14 January 2018

Keywords: F-like ions, energy levels, radiative rates, oscillator strengths, lifetimes

${ }^{*}$ Corresponding author.

Email address: K. Aggarwal@qub.ac.uk (Kanti M. Aggarwal) 


\section{Contents}

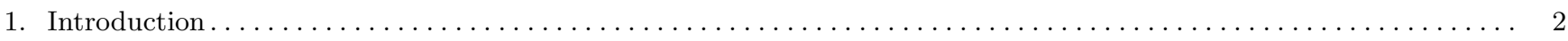

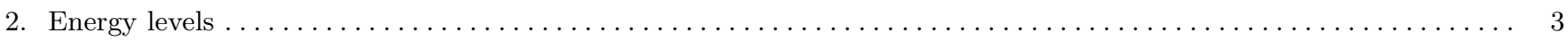

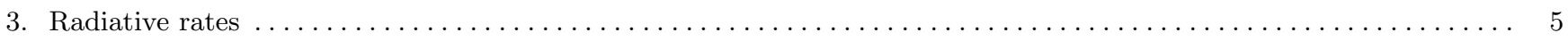

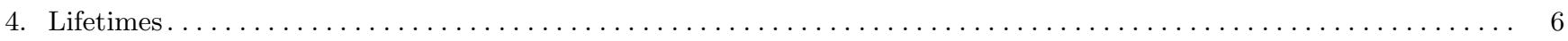

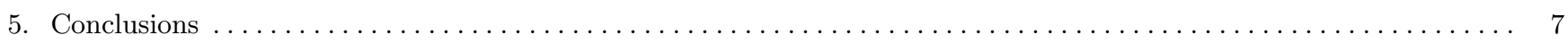

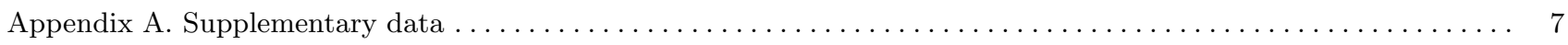

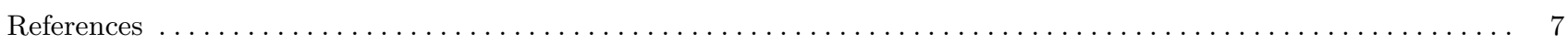

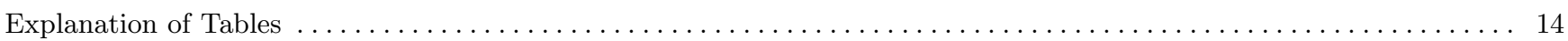

\section{Introduction}

F-like ions have been of interest for the modelling of fusion plasmas for a long time [1], and with the developing ITER project their importance has further increased. The first extensive study for these ions was done by Sampson et al. [2], who performed calculations for a wide range of F-like ions with $22 \leq \mathrm{Z} \leq 92$ by using their Dirac-Fock-Slater (DFS) code. However, for brevity they reported only limited results (and for only a few ions) for energy levels, oscillator strengths (f-values) and collision strengths $(\Omega)$. Unfortunately, most of their data have now been lost. Nevertheless, a few workers after them have performed calculations for a variety of atomic parameters, for a section of these ions. For example, in our earlier study [3], we reported energy levels, radiative rates (A-values), oscillator strengths (f-values), line strengths (S-values), and lifetimes $(\tau)$ for 17 F-like ions with $37 \leq \mathrm{Z} \leq 53$. Similar data for Z $=36$ (Kr XXVIII [4]), 54 (Xe XLVI [5]) and 74 (W LXVI [6-8]) have also been reported. In this paper we list our results for further 19 ions with $55 \leq \mathrm{Z} \leq 73$.

The prior results for these ions, theoretical or experimental, are (mostly) limited to Ba XLVIII alone. Hutton et al. [9] have measured spectra for F-like, O-like and N-like barium ions through the electron beam ion trap (EBIT) machine, and their energy levels have been recommended by the NIST (National Institute of Standards and Technology) team [10], and are available at the website http://www.nist.gov/pml/data/asd.cfm. Similarly, in a laser produced plasma Feldman et al. [11] measured wavelengths for two lines among the lowest three levels of a few F-like ions, and their extrapolated results for Ba XLVIII are also included in the NIST database. Theoretically, as stated earlier Sampson et al. [2] performed calculations for a wide range of F-like ions, but did not specifically report energy levels, and data for other parameters was also limited to a few levels/transitions. Using a combined configuration interaction (CI) and many-body perturbation theory (MBPT) approach, Gu [12] calculated energies for the lowest three levels of F-like ions with $\mathrm{Z} \leq 60$. For the same three levels, later on Jönsson et al. [13] reported energies and A-values for a wider range of ions with $14 \leq \mathrm{Z} \leq 74$, for which they adopted the general-purpose relativistic atomic structure package (GRASP) code [14], and included very large CI for the calculations. Using the original version of the same code (GRASP0 [15]), but extensively modified by (one of the authors) P. H. Norrington, Khatri et al. [16] calculated energies for 431 levels of 
Ba XLVIII, which belong to the 29 configurations, namely $2 \mathrm{~s}^{2} 2 \mathrm{p}^{5}, 2 \mathrm{~s} 2 \mathrm{p}^{6}, 2 \mathrm{~s}^{2} 2 \mathrm{p}^{4} 3 \ell, 2 \mathrm{~s} 2 \mathrm{p}^{5} 3 \ell, 2 \mathrm{p}^{6} 3 \ell, 2 \mathrm{~s}^{2} 2 \mathrm{p}^{4} 4 \ell, 2 \mathrm{~s} 2 \mathrm{p}^{5} 4 \ell$, $2 \mathrm{~s}^{2} 2 \mathrm{p}^{4} 5 \ell$, and $2 \mathrm{~s} 2 \mathrm{p}^{5} 5 \ell$. However, their corresponding results for A-values are limited for transitions from the lowest three levels alone, whereas in a plasma modelling calculation a complete set of data, for all transitions, is preferred. Therefore, their is scope for the extension of their work.

With the easy and free availability of the atomic structure codes and comparatively cheaper access to computational resources, it has become much easier to generate atomic data for various parameters. Unfortunately, producing reliable data with (some measure of) accuracy is still not straightforward because many checks are required before having confidence in the reported data. For energy levels, one may assess the accuracy by: (i) comparing results with two different independent codes, (ii) confirming convergence of results by increasing CI, (iii) comparing results with earlier available theoretical data, and (iv) making comparisons with measurements. In spite of such checks and balances, large discrepancies are often observed for (almost) all atomic parameters, as recently highlighted and explained in our paper [17]. For example, Goyal et al. [18] reported energies for 113 levels of the $2 \mathrm{~s}^{2} 2 \mathrm{p}^{5}, 2 \mathrm{~s} 2 \mathrm{p}^{6}, 2 \mathrm{~s}^{2} 2 \mathrm{p}^{4} 3 \ell, 2 \mathrm{~s} 2 \mathrm{p}^{5} 3 \ell$, and $2 \mathrm{p}^{6} 3 \ell$ configurations of W LXVI, for which they adopted two different codes, namely GRASP and FAC, the flexible atomic code of $\mathrm{Gu}$ [19]. However, for the highest 20 levels the two sets of energies differed by up to $\sim 60$ Ryd. Through our independent calculations [8] it turned out that their results with FAC are incorrect. Similarly, for some Ne-like ions differences in energies, between the GRASP and FAC results, for some levels are up to $\sim 2$ Ryd - see tables 2-4 of [20]. Since such a large difference between any two codes is generally neither noted nor expected, one of the two calculations is actually not correct, as explained in our paper [21]. Therefore, comparison of results with two different codes is only beneficial when both calculations have been performed with great care.

In most atomic structure calculations, inclusion of CI helps to improve the energy levels, and this is particularly true for ions with comparatively lower atomic numbers. However, there is always a limit up to which the calculations can be performed, and in many cases inclusion of CI beyond a (certain) level is of no real advantage. For F-like ions an extensive inclusion of CI is not very beneficial, as noted in our earlier work [3], although Jönsson et al. [13] have performed very large calculations by including up to 73000 and $15000 \mathrm{CSFs}$ (configuration state functions) for the $2 \mathrm{~s}^{2} 2 \mathrm{p}^{5}{ }^{2} \mathrm{P}_{1 / 2}^{o}$ and $2 \mathrm{~s} 2 \mathrm{p}^{6}{ }^{2} \mathrm{~S}_{1 / 2}$ levels, as a result of which their energies closely match with the measurements. However, inclusion of such a large $\mathrm{CI}$ is not practical for a large number of levels (and ions), particularly when measurements are almost non existent.

Comparison of results with existing data are always useful and considerably help in improving the calculations - see for example the recent work of Guo et al. [22] on W XL and the references within the paper. However, the problem arises when no prior data exist as noted for some of the Cr-like ions [23]. Similarly, if measurements are available, even for a few levels, it considerably helps to improve the calculations, as was the case for some other Cr-like ions - see Table A of [24]. Unfortunately, for the current F-like ions of interest, theoretical data are (mostly) restricted to the lowest three levels, and the measurements for a few levels are available for only Ba XLVIII. Therefore, we will make most of the comparisons for this ion alone to make some assessment of the accuracy of our calculated data.

\section{Energy levels}

As in our earlier work [3], we adopt the GRASP0 version which is hosted at the website http://amdpp.phys.strath.ac.uk/UK For the optimisation of the orbitals we use the option of 'extended average level' (EAL), in which a weighted (proportional to $2 j+1$ ) trace of the Hamiltonian matrix is minimised. Additionally, the contributions of Breit and quantum 
electrodynamic effects (QED) are included, which are important for the heavy ions considered in this work. Calculations for energy levels and subsequent other parameters have been performed among 501 levels of 38 configurations, namely $2 \mathrm{~s}^{2} 2 \mathrm{p}^{5}, 2 \mathrm{~s} 2 \mathrm{p}^{6}, 2 \mathrm{~s}^{2} 2 \mathrm{p}^{4} 3 \ell, 2 \mathrm{~s} 2 \mathrm{p}^{5} 3 \ell, 2 \mathrm{p}^{6} 3 \ell, 2 \mathrm{~s}^{2} 2 \mathrm{p}^{4} 4 \ell, 2 \mathrm{~s} 2 \mathrm{p}^{5} 4 \ell, 2 \mathrm{p}^{6} 4 \ell, 2 \mathrm{~s}^{2} 2 \mathrm{p}^{4} 5 \ell, 2 \mathrm{~s} 2 \mathrm{p}^{5} 5 \ell$, and $2 \mathrm{p}^{6} 5 \ell$. However, as in the past, our focus is on 113 levels of the $2 \mathrm{~s}^{2} 2 \mathrm{p}^{5}, 2 \mathrm{~s} 2 \mathrm{p}^{6}, 2 \mathrm{~s}^{2} 2 \mathrm{p}^{4} 3 \ell, 2 \mathrm{~s} 2 \mathrm{p}^{5} 3 \ell$, and $2 \mathrm{p}^{6} 3 \ell$ (11) configurations. Furthermore, A-values have been calculated for four types of transitions, namely electric dipole (E1), magnetic dipole (M1), electric quadrupole (E2), and magnetic quadrupole (M2). These results are required for the further calculation of lifetimes as well as in plasma modelling.

Our calculated energies with GRASP for all 113 levels for F-like ions with $55 \leq \mathrm{Z} \leq 73$ are listed in Tables 1-19. For the ground level $\left(2 \mathrm{~s}^{2} 2 \mathrm{p}^{5}{ }^{2} \mathrm{P}_{3 / 2}^{o}\right)$ absolute energies are listed whereas for others are differences w.r.t. to the ground. Since there is a paucity of prior theoretical and experimental data for these ions, we have performed analogous calculations with FAC (https://www-amdis.iaea.org/FAC/), which is also a fully relativistic code and generally yields energy levels of comparable accuracy, but with much more efficiency. With this code three calculations have been performed which are: (i) FAC1, which includes 501 levels of the the same configurations as included with GRASP, (ii) FAC2, which includes 38089 levels arising from all possible combinations of the $\left(2^{*} 5\right) 3^{*} 2,4^{*} 2,5^{*} 2,3^{*} 14^{*} 1,3^{*} 15^{*} 1$, and $4^{*} 15^{*} 1$ configurations, plus those of FAC1, and (iii) FAC3, which includes a total of 72259 levels, the additional ones arising from the $\left(2^{*} 6\right) 6^{*} 1,7^{*} 1,8^{*} 1$ and $\left(2^{*} 53^{*} 1\right) 6^{*} 1,7^{*} 1$, and $8^{*} 1$ configurations. Since our FAC1 energies are almost comparable with those with GRASP, in both magnitude and orderings, we list these only for Ba XLVIII in Table 2. This result was expected because both calculations include the same CI. However, our FAC2 calculations include much more extensive CI and therefore are expected to be more accurate, we include these results for all ions. As was the case earlier for other F-like ions [3], the FAC3 calculations offer no real advantage, because there are no appreciable differences with the FAC2 energies, for the lowest 113 levels under consideration. For this reason we do not discuss the FAC3 energies any further. Similarly, the results of Khatri et al. [16] are not included in Table 2 because there are no differences with our present calculations. This is mainly because both calculations adopt the same code and the addition of further 70 levels of the $2 \mathrm{p}^{6} 4 \ell$ and $2 \mathrm{p}^{6} 5 \ell(9)$ configurations in our calculations has an insignificant effect on the 113 levels considered in this work.

As already stated, the contributions of Breit and QED effects are very important and significant for the determination of energy levels for these heavy ions. However, the maximum effect is on the ground levels and therefore, in Table A along with the Coulomb energies we list contributions of Breit and QED corrections for all ions. Although the combined effect of these corrections on total energies is less than $0.2 \%$, in absolute terms these are very significant. For example, for Cs XLVII their respective contributions amount to $9.1+7.6=16.7 \mathrm{Ryd}$, but increase by a factor of 2.5 to $22.7+20.0$ $=42.7$ Ryd for Ta LXV. In comparison, the maximum combined contribution to other levels is $\sim 4$ Ryd, i.e. less than 10\%. Similarly, differences between the absolute energies obtained for the ground level with GRASP and FAC increase with increasing $\mathrm{Z}$, as it is only $\sim 1$ Ryd for Cs XLVII but $\sim 6$ Ryd for Ta LXV, and energies with the former are always the lowest. However, comparative differences between energies from both calculations remain within $\sim 0.5 \mathrm{Ryd}$, for all excited levels and ions, as seen in Tables 1-19.

Before we discuss our results in detail, we would like to emphasize on two points. Firstly, the level designations provided in Tables 1-19 may not be unambiguous. This is because some of the levels are highly mixed, and for a few mixing coefficient from a particular level/configuration may dominate in more than one levels. This is a general atomic 
structure problem, irrespective of the code adopted. Although this has been discussed several times in some of our earlier papers (as well as by other workers), in Table B we provide the mixing coefficients for the levels of Cs XLVII, to give some idea of the problem. Among the problematic levels are 12/35, 15/44 and 18/47. We have tried to provide a unique designation for each level, but that is subject to change with other calculations and/or workers. Therefore, only the $J^{\pi}$ values should be considered definite. Secondly, unlike the other F-like ions considered in our earlier work [3], the 113 levels listed in the tables are lowest for Cs XLVII alone. For other ions, some from other configurations, mainly $2 \mathrm{~s}^{2} 2 \mathrm{p}^{4} 4 \ell$, intermix. To consider all listed levels of Tables 1-19 in strictly increasing order of energy, we have to consider up to 147 levels, although it varies from ion to ion. However, irrespective of the ion the first 94 levels are the lowest in energy.

In Table C we compare our theoretical energies with GRASP and FAC codes, obtained with 501 and 38089 levels, respectively, with the measurements of Hutton et al. [9] for the common levels of Ba XLVIII, which have been recommended by the NIST team. There are no large discrepancies for these levels, although differences are up to $\sim 0.4$ Ryd for a few, such as $2 \mathrm{~s} 2 \mathrm{p}^{6}{ }^{2} \mathrm{~S}_{1 / 2}(3), 2 \mathrm{~s}^{2} 2 \mathrm{p}^{4}\left({ }^{3} \mathrm{P}\right) 3 \mathrm{~d} \quad{ }^{2} \mathrm{P}_{1 / 2}(9), 2 \mathrm{~s}^{2} 2 \mathrm{p}^{4}\left({ }^{3} \mathrm{P}\right) 3 \mathrm{~d} \quad{ }^{2} \mathrm{P}_{3 / 2}(11)$, and $2 \mathrm{~s}^{2} 2 \mathrm{p}^{4}\left({ }^{1} \mathrm{~S}\right) 3 \mathrm{~d} \quad{ }^{2} \mathrm{D}_{5 / 2} \quad(12)$. Furthermore, these differences are not systematic as for level 3 theoretical results are higher, but lower for others. In general, GRASP and FAC energies agree within 0.1 Ryd, the latter being mostly lower, and hence the agreement of NIST is slightly better with those with GRASP. We will also like to note here that the energy for the level $3\left(2 \mathrm{~s} 2 \mathrm{p}^{6}{ }^{2} \mathrm{~S}_{1 / 2}\right)$ is not based on direct measurement, although Jönsson et al. [13] have been able to reproduce it with extensive CI, as discussed below.

In Table D we compare our energies calculated with the GRASP and FAC codes with those of Jönsson et al. [13] for the two (common) levels $2 \mathrm{~s}^{2} 2 \mathrm{p}^{5}{ }^{2} \mathrm{P}_{1 / 2}^{o}$ and $2 \mathrm{~s} 2 \mathrm{p}^{6}{ }^{2} \mathrm{~S}_{1 / 2}$, for all ions. Also included in this table are the earlier energies of $\mathrm{Gu}$ [12], obtained with the CI+MBPT code for the lowest six ions. Differences between the GRASP and FAC calculations, or with the results of $\mathrm{Gu}$, are not significant and are within $\sim 0.2$ Ryd for both levels. Similarly, there is a good agreement with the results of Jönsson et al. for the ${ }^{2} \mathrm{P}_{1 / 2}^{o}$ level, but discrepancies for ${ }^{2} \mathrm{~S}_{1 / 2}$ are comparatively larger, and increase with increasing Z, as these are 0.2 Ryd for Cs XLVII but 0.5 Ryd for Ta LXV, with our results being invariably higher. Since the calculations of Jönsson et al. are comparatively more sophisticated, we believe these to be more accurate as well. Therefore, based on this comparison as well as the one discussed earlier, we may state that our energy levels are accurate to $\sim 0.5 \mathrm{Ryd}$. This conclusion is also based on comparisons between the GRASP and FAC energies for a larger number of levels - see Tables 1-19. However, we emphasize again that differences between the two calculations are not systematic, as for some the GRASP energies are higher whereas the reverse is true for the FAC see for example, levels 64 and 80 of Ba XLVIII in Table 2. Therefore, inclusion of larger CI does not necessarily produce lower energies for all levels.

\section{Radiative rates}

Our calculated results with the GRASP code are listed in Tables 20-38 for transition energies (wavelengths, $\lambda_{j i}$ in $\AA$ ), radiative rates (A-values, in $\mathrm{s}^{-1}$ ), oscillator strengths (f-values, dimensionless), and line strengths (S-values, in atomic units, 1 a.u. $=6.460 \times 10^{-36} \mathrm{~cm}^{2} \mathrm{esu}^{2}$ ) for $\mathrm{E} 1$ transitions in F-like ions with $55 \leq \mathrm{Z} \leq 73$. These results are obtained in both the velocity (Coulomb gauge) and length (Babushkin gauge) forms. Ideally both forms should give comparable results with their ratio $(\mathrm{R})$ close(r) to unity, but in practice they may differ substantially, especially for the very weak 
transitions with very small f-values. Nevertheless, we have also listed $\mathrm{R}$ in these tables for all E1 transitions, which are the most significant in any calculation. Similar results for E2, M1 and M2 transitions are listed only for the A-values, because the corresponding data for f- or S-values can be obtained using Eqs. (1-5) given in [4]. Finally, the indices used to represent the lower and upper levels of a transition are defined in Tables 1-19. Furthermore, for brevity only transitions from the lowest 3 to higher excited levels are listed in Tables 20-38, but full tables in the ASCII format are available online in the electronic version.

The only results for A-values available for the comparison purpose are those of Khatri et al. [16] for transitions from the lowest three to higher excited levels of Ba XLVIII. With them we have no differences for the same reason as for the energy levels. However, Jönsson et al. [13] have reported A-values for four transitions, namely 1-3 E1, 2-3 E1, 1-2 M1, and 1-2 E2, but for all F-like ions of interest. Therefore, in Table E we make comparison between our and their results. It is highly satisfactory to note that there is no discrepancy for any transition and/or ion, and all results agree to better than $5 \%$. This is in spite of some differences in energies, particularly for the $2 \mathrm{~s} 2 \mathrm{p}^{6}{ }^{2} \mathrm{~S}_{1 / 2}$ level, as seen earlier in Table D. This confirms (yet again) that small differences in transition energies do not lead to any appreciable differences in the subsequent results for A-values. In conclusion, based on our experience and comparisons made earlier for other F-like ions [3], our assessment of accuracy for the f- (and A-) values for a majority of strong transitions is $\sim 20 \%$, for all ions.

Some times A-values for E3 (electric octupole) transitions may also be useful, if their strengths are comparable to others, such as the M2. However, for the F-like ions under consideration this is not the case. For example, for Cs XLVII there are 2299 possible E3 transitions among the 113 levels, but only 10 of these have $\mathrm{f} \sim 10^{-6}$ and the rest are much weaker. On the other hand, there are $5 \mathrm{M} 2$ transitions with $\mathrm{f} \sim 10^{-5}$, i.e. the E3 transitions are weaker than M2 by at least an order of magnitude. Similarly, for Gd LVI there are 19 E3 and 10 M2 such transitions with $\mathrm{f} \sim 10^{-6}$ and $\mathrm{f} \sim 10^{-5}$, respectively. Finally, for Ta LXV there are $19 \mathrm{E} 3$ and $13 \mathrm{M} 2$ such transitions with $\mathrm{f} \sim 10^{-6}$ and $\mathrm{f} \sim 10^{-5}$, respectively. Therefore, we have not included A-values for E3 transitions in Tables 20-38 but the results can be obtained from the author on request.

\section{Lifetimes}

The lifetime $\tau$ of a level $j$ is related to the A-values as 1.0/ $\Sigma_{i} \mathrm{~A}_{j i}$. As stated earlier, E1 transitions are (normally) the most dominant, and hence important in the determination of $\tau$. However, summation over all types of transitions, i.e. $\mathrm{E} 1, \mathrm{E} 2, \mathrm{M} 1$, and M2, improves the accuracy and is particularly important for those levels for which there are no (strong) E1 connections. Although $\tau$ is a measurable quantity, no experiments have yet been performed for transitions/levels of F-like ions of present interest. Therefore, no hard assessments of accuracy can be made. Unfortunately, the situation is no better with the theory. However, Khatri et al. [16] have listed $\tau$ for the levels of Ba XLVIII for which we have no differences, except for level 3, i.e. $2 \mathrm{~s} 2 \mathrm{p}^{6}{ }^{2} \mathrm{~S}_{1 / 2}$. For this level their listed $\tau$ is $1.43 \times 10^{-13} \mathrm{~s}$, whereas our result is $4.11 \times 10^{-13} \mathrm{~s}$, larger by a factor of three. Their result for this level is incorrect, because the dominant contributing transition for this is $1-3 \mathrm{E} 1$ for which their A-value is $2.34 \times 10^{12} \mathrm{~s}^{-1}$, which leads to $\tau=4.27 \times 10^{-13} \mathrm{~s}$, closer to our calculation. A similar discrepancy was noted [25] in their results for the $2 \mathrm{~s}^{2} 2 \mathrm{p}^{5}{ }^{2} \mathrm{P}_{1 / 2}^{o}$ level of Sr XXX. For future comparisons with experimental or theoretical data our calculated values of $\tau$ are included in Tables 1-19. Since these results are directly related to the A-values, our assessment of accuracy for these is also the same, i.e. $\sim 20 \%$. 


\section{Conclusions}

In this paper, energies for 113 levels of the $2 \mathrm{~s}^{2} 2 \mathrm{p}^{5}, 2 \mathrm{~s} 2 \mathrm{p}^{6}, 2 \mathrm{~s}^{2} 2 \mathrm{p}^{4} 3 \ell, 2 \mathrm{~s} 2 \mathrm{p}^{5} 3 \ell$, and $2 \mathrm{p}^{6} 3 \ell$ configurations of $19 \mathrm{~F}-\mathrm{like}$ ions with $55 \leq \mathrm{Z} \leq 73$ are reported. Combined with our earlier results [3-5], this presents a complete data for ions with $\mathrm{Z} \leq 74$. Similarly as earlier, we have adopted the GRASP code for the calculations. Since no existing data are available for most of the levels and ions with which to make comparisons, we have made additional calculations with the FAC code, but with much more extensive CI. This helps in assessing the accuracy of the energy levels. Based on several calculations with both codes, as well as comparisons with available limited theoretical and experimental data, our energy levels are assessed to be accurate to better than $0.5 \%$ (0.5 Ryd), for all ions. However, for a few levels of each ion there is some ambiguity in their designations. This is because of very strong mixing with one eigenvector of a CSF often dominating in magnitude for several levels. For this reason, mixing coefficients are listed for the levels of Cs XLVII, as an example. However, similar results for other ions can be obtained from the author on request.

Radiative rates for four types of transitions, i.e. E1, E2, M1, and M2, are also reported among the above listed 113 levels. These data are significantly more extensive than currently available in the literature. Comparisons with the existing literature are limited to only four transitions, for which there are no discrepancies. Similarly, calculations for comparatively larger number of transitions for Ba XLVIII are also available. Again, there are no discrepancies with our results because of the inclusion of similar level of CI and code. Based on whatever comparisons are possible and with our past experience on calculations for a wide range of ions, we assess our A-values and lifetimes to be accurate to $\sim 20 \%$, particularly for strong transitions with large f-values. For very weak transitions the reported A-values may be comparatively less reliable.

\section{Appendix A. Supplementary data}

Owing to space limitations, only parts of Tables 20-38 are presented here, but full tables are being made available as supplemental material in conjunction with the electronic publication of this work. Supplementary data associated with this article can be found, in the online version, at doi:nn.nnnn/j.adt.2018.nn.nnn.

\section{References}

\section{References}

[1] P.L. Hagelstein, Phys. Rev. A 34 (1986) 924.

[2] D.H. Sampson, H.L. Zhang, C.J. Fontes, At. Data Nucl. Data Tables 48 (1991) 25.

[3] K.M. Aggarwal, F.P. Keenan, At. Data Nucl. Data Tables 109-110 (2016) 205.

[4] K.M. Aggarwal, F.P. Keenan, K.D. Lawson, At. Data Nucl. Data Tables 94 (2008) 323.

[5] K.M. Aggarwal, F.P. Keenan, K.D. Lawson, At. Data Nucl. Data Tables 96 (2010) 123.

[6] K.M. Aggarwal, F.P. Keenan, At. Data Nucl. Data Tables 111-112 (2016) 187.

[7] K.M. Aggarwal, Chin. Phys. B 25 (2016) 043201.

[8] K.M. Aggarwal, Atoms 5 (2016) 4030024. 
[9] R. Hutton, P. Beiersdorfer, A.L. Osterheld, R.E. Marrs, M.B. Schneider, Phys. Rev. A 44 (1991) 1836.

[10] J.E. Sansonetti, J.J. Curry, J. Phys. Chem. Ref. Data 39 (2010) 043103.

[11] U. Feldman, J.O. Ekberg, J.F. Seely, C.M. Brown, D.R. Kania, B.J. MacGowan, C.J. Keane, W.E. Behring, J. Opt. Soc. Am. B 8 (1991) 531.

[12] M.F. Gu, At. Data Nucl. Data Tables 85 (2005) 267.

[13] P. Jönsson, A. Alkauskas, G. Gaigalas, At. Data Nucl. Data Tables 99 (2013) 431.

[14] P. Jönsson, X. He, C.F. Fischer, I.P. Grant, Comput. Phys. Commun. 177 (2007) 597.

[15] I.P. Grant, B.J. McKenzie, P.H. Norrington, D.F. Mayers, N.C. Pyper, Comput. Phys. Commun. 21 (1980) 207.

[16] I. Khatri, A. Goyal, S. Aggarwal, A.K. Singh, M. Mohan, At. Data Nucl. Data Tables 107 (2016) 367.

[17] K.M. Aggarwal, Atoms 5 (2017) 5040037.

[18] A. Goyal, I. Khatri, S. Aggarwal, A.K. Singh, R. Sharma, M. Mohan, J. At. Mol. Cond. Nano Phys. 2 ( 2015 ) 1.

[19] M.F. Gu, Can. J. Phys. 86 (2008) 675.

[20] N. Singh, S. Aggarwal, M. Mohan, Can. J. Phys. 96 (2018) 36.

[21] K.M. Aggarwal, Can. J. Phys. 96 (2018) - to be submitted.

[22] X.L. Guo, M.C. Li, R. Si, X.D. He, K. Wang, Z.T. Dai, Y.M. Liu, H.J. Zhang, C.Y. Chen, J. Phys. B 51 (2018) 015002 .

[23] K.M. Aggarwal, P. Bogdanovich, R. Karpuškienè, F.P. Keenan, R. Kisielius, At. Data Nucl. Data Tables 120 (2018) in press.

[24] K.M. Aggarwal, P. Bogdanovich, R. Karpuškienè, F.P. Keenan, R. Kisielius, V. Stancalie, At. Data Nucl. Data Tables 107 (2016) 140.

[25] K.M. Aggarwal, J. Quant. Spect. Rad. Transf. 166 (2015) 108. 
Table A

Ground level $\left(2 \mathrm{~s}^{2} 2 \mathrm{p}^{5}{ }^{2} \mathrm{P}_{3 / 2}^{o}\right)$ energies (in Ryd) from the GRASP calculations with contributions from Breit and QED effects for F-like ions with $55<\mathrm{Z}<73$.

\begin{tabular}{llllll}
\hline $\mathrm{Z}$ & Ion & Coulomb & Breit & QED & Total \\
\hline 55 & Cs XLVII & -10995.70 & 09.09 & 07.56 & -10979.05 \\
56 & Ba XLVII & -11430.84 & 09.63 & 08.04 & -11413.17 \\
57 & La XLIX & -11875.54 & 10.2 & 08.54 & -11856.80 \\
58 & Ce L & -12329.87 & 10.8 & 09.06 & -12310.03 \\
59 & Pr LI & -12793.94 & 11.4 & 09.60 & -12772.95 \\
60 & Nd LII & -13267.86 & 12.0 & 10.2 & -13245.67 \\
61 & Pm LII & -13751.73 & 12.7 & 10.8 & -13728.29 \\
62 & Sm LIV & -14245.65 & 13.4 & 11.4 & -14220.92 \\
63 & Eu LV & -14749.75 & 14.1 & 12.0 & -14723.66 \\
64 & Gd LVI & -15264.14 & 14.8 & 12.7 & -15236.64 \\
65 & Tb LVII & -15788.93 & 15.6 & 13.4 & -15759.98 \\
66 & Dy LVIII & -16324.27 & 16.3 & 14.1 & -16293.80 \\
67 & Ho LIX & -16870.27 & 17.2 & 14.9 & -16838.24 \\
68 & Er LX & -17427.08 & 18.0 & 15.7 & -17393.41 \\
69 & Tm LXI & -17994.83 & 18.9 & 16.5 & -17959.48 \\
70 & Yb LXII & -18573.68 & 19.8 & 17.3 & -18536.58 \\
71 & Lu LXIII & -19163.78 & 20.7 & 18.2 & -19124.86 \\
72 & Hf LXIV & -19765.28 & 21.7 & 19.1 & -19724.48 \\
73 & Ta LXV & -20378.36 & 22.7 & 20.0 & -20335.61 \\
\hline
\end{tabular}


Table B

Mixing coefficients (MC) for 113 levels of Cs XLVII. Numbers outside and inside a bracket correspond to MC and the level, respectively.

\begin{tabular}{|c|c|c|c|}
\hline Index & Configuration & Level & Mixing coefficients \\
\hline 1 & $2 \mathrm{~s}^{2} 2 \mathrm{p}^{5}$ & ${ }^{2} \mathrm{P}_{3 / 2}^{o}$ & $1.00(1)$ \\
\hline 2 & $2 \mathrm{~s}^{2} 2 \mathrm{p}^{5}$ & ${ }^{2} \mathrm{P}_{1 / 2}^{o}$ & $1.00(2)$ \\
\hline 3 & $2 \mathrm{~s} 2 \mathrm{p}^{6}$ & ${ }^{2} \mathrm{~S}_{1 / 2}$ & $1.00(3)$ \\
\hline 4 & $2 \mathrm{~s}^{2} 2 \mathrm{p}^{4} 3 \mathrm{~s}$ & ${ }^{4} \mathrm{P}_{5 / 2}$ & $0.84(4)+0.54(28)$ \\
\hline 5 & $2 \mathrm{~s}^{2} 2 \mathrm{p}^{4} 3 \mathrm{~s}$ & ${ }^{2} \mathrm{P}_{3 / 2}$ & $0.35(26)+0.75(5)+0.55(29)$ \\
\hline 6 & $2 \mathrm{~s}^{2} 2 \mathrm{p}^{4} 3 \mathrm{~s}$ & ${ }^{2} \mathrm{~S}_{1 / 2}$ & $0.51(62)-0.34(27)+0.79(6)$ \\
\hline 7 & $2 \mathrm{~s}^{2} 2 \mathrm{p}^{4}\left({ }^{3} \mathrm{P}\right) 3 \mathrm{p}$ & ${ }^{4} \mathrm{P}_{3 / 2}^{o}$ & $-0.27(31)+0.58(7)-0.43(13)+0.30(14)+0.37(37)-0.40(33)$ \\
\hline 8 & $2 \mathrm{~s}^{2} 2 \mathrm{p}^{4}\left({ }^{3} \mathrm{P}\right) 3 \mathrm{p}$ & ${ }^{2} \mathrm{D}_{5 / 2}^{3 / 2}$ & $0.53(34)-0.36(10)+0.54(8)+0.48(32)-0.26(39)$ \\
\hline 9 & $2 \mathrm{~s}^{2} 2 \mathrm{p}^{4}\left({ }^{1} \mathrm{~S}\right) 3 \mathrm{p}$ & ${ }^{2} \mathrm{P}_{1 / 2}^{\mathrm{o} / 2}$ & $0.44(69)+0.24(30)+0.33(12)+0.78(9)$ \\
\hline 10 & $2 \mathrm{~s}^{2} 2 \mathrm{p}^{4}\left({ }^{3} \mathrm{P}\right) 3 \mathrm{p}$ & ${ }^{4} \mathrm{P}_{5 / 2}^{o}$ & $0.64(10)+0.53(8)+0.28(32)+0.46(39)$ \\
\hline 11 & $2 \mathrm{~s}^{2} 2 \mathrm{p}^{4}\left({ }^{3} \mathrm{P}\right) 3 \mathrm{p}$ & ${ }^{4} \mathrm{D}_{7 / 2}^{5 / 2}$ & $0.84(11)+0.55(38)$ \\
\hline 12 & $2 \mathrm{~s}^{2} 2 \mathrm{p}^{4}\left({ }^{3} \mathrm{P}\right) 3 \mathrm{p}$ & ${ }^{2} \mathrm{P}_{1 / 2}^{o}$ & $0.20(69)-0.29(30)-0.40(12)+0.62(35)+0.56(40)$ \\
\hline 13 & $2 \mathrm{~s}^{2} 2 \mathrm{p}^{4}\left({ }^{3} \mathrm{P}\right) 3 \mathrm{p}$ & ${ }^{4} \mathrm{~S}_{3 / 2}^{\mathrm{o}}$ & $0.51(13)-0.40(36)+0.46(14)+0.37(37)+0.32(33)+0.36(78)$ \\
\hline 14 & $2 \mathrm{~s}^{2} 2 \mathrm{p}^{4}\left({ }^{3} \mathrm{P}\right) 3 \mathrm{p}$ & ${ }^{2} \mathrm{P}_{3 / 2}^{0}$ & $0.40(31)+0.35(7)-0.38(14)-0.22(37)+0.70(78)$ \\
\hline 15 & $2 \mathrm{~s}^{2} 2 \mathrm{p}^{4}\left({ }^{3} \mathrm{P}\right) 3 \mathrm{~d}$ & ${ }^{4} \mathrm{D}_{3 / 2}^{3 / 2}$ & $-0.58(15)+0.47(24)-0.27(58)+0.46(44)-0.28(52)$ \\
\hline 16 & $2 \mathrm{~s}^{2} 2 \mathrm{p}^{4}\left({ }^{3} \mathrm{P}\right) 3 \mathrm{~d}$ & ${ }^{4} \mathrm{D}_{5 / 2}$ & $-0.34(45)+0.64(16)-0.28(93)+0.26(23)+0.41(51)-0.34(56)$ \\
\hline 17 & $2 \mathrm{~s}^{2} 2 \mathrm{p}^{4}\left({ }^{3} \mathrm{P}\right) 3 \mathrm{~d}$ & ${ }^{4} \mathrm{P}_{1 / 2}$ & $0.39(42)-0.67(17)-0.32(21)+0.50(60)-0.24(48)$ \\
\hline 18 & $2 \mathrm{~s}^{2} 2 \mathrm{p}^{4}\left({ }^{3} \mathrm{P}\right) 3 \mathrm{~d}$ & ${ }^{2} \mathrm{~F}_{7 / 2}$ & $0.56(47)-0.30(19)+0.54(18)+0.50(46)-0.22(57)$ \\
\hline 19 & $2 \mathrm{~s}^{2} 2 \mathrm{p}^{4}\left({ }^{3} \mathrm{P}\right) 3 \mathrm{~d}$ & ${ }^{4} \mathrm{D}_{7 / 2}$ & $0.63(19)+0.52(18)+0.24(46)+0.48(57)$ \\
\hline 20 & $2 \mathrm{~s}^{2} 2 \mathrm{p}^{4}\left({ }^{3} \mathrm{P}\right) 3 \mathrm{~d}$ & ${ }^{4} \mathrm{~F}_{9 / 2}$ & $0.83(20)+0.55(53)$ \\
\hline 21 & $2 \mathrm{~s}^{2} 2 \mathrm{p}^{4}\left({ }^{3} \mathrm{P}\right) 3 \mathrm{~d}$ & ${ }^{2} \mathrm{P}_{1 / 2}$ & $0.45(17)-0.67(21)+0.27(60)+0.49(48)$ \\
\hline 22 & $2 \mathrm{~s}^{2} 2 \mathrm{p}^{4}\left({ }^{1} \mathrm{~S}\right) 3 \mathrm{~d}$ & ${ }^{2} \mathrm{D}_{3 / 2}$ & $0.32(92)+0.41(24)+0.41(58)-0.28(52)+0.67(22)$ \\
\hline 23 & $2 \mathrm{~s}^{2} 2 \mathrm{p}^{4}\left({ }^{3} \mathrm{P}\right) 3 \mathrm{~d}$ & ${ }^{2} \mathrm{D}_{5 / 2}$ & $0.46(93)-0.40(49)+0.53(23)+0.34(51)+0.40(56)$ \\
\hline 24 & $2 \mathrm{~s}^{2} 2 \mathrm{p}^{4}\left({ }^{3} \mathrm{P}\right) 3 \mathrm{~d}$ & ${ }^{4} \mathrm{P}_{3 / 2}$ & $0.34(92)-0.31(24)-0.30(58)+0.53(50)+0.30(44)+0.36(52)+0.40(22)$ \\
\hline 25 & $2 \mathrm{~s}^{2} 2 \mathrm{p}^{4}\left({ }^{1} \mathrm{~S}\right) 3 \mathrm{~d}$ & ${ }^{2} \mathrm{D}_{5 / 2}$ & $0.35(45)+0.31(16)-0.29(23)+0.75(25)$ \\
\hline 26 & $2 \mathrm{~s}^{2} 2 \mathrm{p}^{4} 3 \mathrm{~s}$ & ${ }^{4} \mathrm{P}_{3 / 2}$ & $0.91(26)-0.34(5)$ \\
\hline 27 & $2 \mathrm{~s}^{2} 2 \mathrm{p}^{4} 3 \mathrm{~s}$ & ${ }^{2} \mathrm{P}_{1 / 2}$ & $0.58(62)+0.81(27)$ \\
\hline 28 & $2 s^{2} 2 p^{4} 3 s$ & ${ }^{2} \mathrm{D}_{5 / 2}$ & $0.53(4)-0.82(28)$ \\
\hline 29 & $2 \mathrm{~s}^{2} 2 \mathrm{p}^{4} 3 \mathrm{~s}$ & ${ }^{2} \mathrm{D}_{3 / 2}$ & $0.55(5)-0.82(29)$ \\
\hline 30 & $2 s^{2} 2 p^{4}\left({ }^{3} P\right) 3 p$ & ${ }^{4} \mathrm{P}_{1 / 2}^{o}$ & $-0.51(69)+0.73(30)+0.45(35)$ \\
\hline 31 & $2 \mathrm{~s}^{2} 2 \mathrm{p}^{4}\left({ }^{3} \mathrm{P}\right) 3 \mathrm{p}$ & ${ }^{4} \mathrm{D}_{3 / 2}^{1 / 2}$ & $0.73(31)+0.36(36)+0.39(14)+0.25(37)-0.28(33)$ \\
\hline 32 & $2 \mathrm{~s}^{2} 2 \mathrm{p}^{4}\left({ }^{1} \mathrm{D}\right) 3 \mathrm{p}$ & ${ }^{2} \mathrm{~F}_{5 / 2}^{o}$ & $0.33(34)-0.21(10)+0.38(8)-0.74(32)+0.40(39)$ \\
\hline 33 & $2 \mathrm{~s}^{2} 2 \mathrm{p}^{4}\left({ }^{1} \mathrm{D}\right) 3 \mathrm{p}$ & ${ }^{2} \mathrm{P}_{3 / 2}^{5 / 2}$ & $0.33(7)-0.32(13)+0.44(14)-0.46(37)+0.59(33)$ \\
\hline 34 & $2 \mathrm{~s}^{2} 2 \mathrm{p}^{4}\left({ }^{3} \mathrm{P}\right) 3 \mathrm{p}$ & ${ }^{4} \mathrm{D}_{5 / 2}^{O}$ & $0.74(34)+0.54(10)-0.34(8)$ \\
\hline 35 & $2 \mathrm{~s}^{2} 2 \mathrm{p}^{4}\left({ }^{3} \mathrm{P}\right) 3 \mathrm{p}$ & ${ }^{2} \mathrm{~S}_{1 / 2}^{o}$ & $-0.32(69)-0.50(30)+0.45(12)+0.52(35)-0.40(40)$ \\
\hline 36 & $2 \mathrm{~s}^{2} 2 \mathrm{p}^{4}\left({ }^{3} \mathrm{P}\right) 3 \mathrm{p}$ & ${ }^{2} \mathrm{D}_{3 / 2}^{\mathrm{O}}$ & $0.48(7)+0.51(13)+0.69(36)$ \\
\hline 37 & $2 \mathrm{~s}^{2} 2 \mathrm{p}^{4}\left({ }^{1} \mathrm{D}\right) 3 \mathrm{p}$ & ${ }^{2} \mathrm{D}_{3 / 2}^{o / 2}$ & $0.33(13)+0.41(14)-0.62(37)-0.53(33)$ \\
\hline 38 & $2 \mathrm{~s}^{2} 2 \mathrm{p}^{4}\left({ }^{1} \mathrm{D}\right) 3 \mathrm{p}$ & ${ }^{2} \mathrm{~F}_{7 / 2}^{o / 2}$ & $0.55(11)-0.84(38)$ \\
\hline 39 & $2 \mathrm{~s}^{2} 2 \mathrm{p}^{4}\left({ }^{1} \mathrm{D}\right) 3 \mathrm{p}$ & ${ }^{2} \mathrm{D}_{5 / 2}^{o}$ & $-0.22(34)+0.30(10)+0.41(8)-0.38(32)-0.73(39)$ \\
\hline 40 & $2 \mathrm{~s}^{2} 2 \mathrm{p}^{4}\left({ }^{1} \mathrm{D}\right) 3 \mathrm{p}$ & ${ }^{2} \mathrm{P}_{1 / 2}^{o / 2}$ & $0.30(69)-0.58(12)+0.22(35)-0.70(40)$ \\
\hline 41 & $2 \mathrm{~s} 2 \mathrm{p}^{5}\left({ }^{3} \mathrm{P}\right) 3 \mathrm{~s}$ & ${ }^{4} \mathrm{P}_{5 / 2}^{o}$ & $0.98(41)$ \\
\hline 42 & $2 \mathrm{~s}^{2} 2 \mathrm{p}^{4}\left({ }^{3} \mathrm{P}\right) 3 \mathrm{~d}$ & ${ }^{4} \mathrm{D}_{1 / 2}$ & $-0.90(42)-0.32(21)+0.22(60)$ \\
\hline 43 & $2 \mathrm{~s} 2 \mathrm{p}^{5}\left({ }^{3} \mathrm{P}\right) 3 \mathrm{~s}$ & ${ }^{2} \mathrm{P}_{3 / 2}^{o}$ & $0.51(55)+0.84(43)$ \\
\hline 44 & $2 \mathrm{~s}^{2} 2 \mathrm{p}^{4}\left({ }^{1} \mathrm{D}\right) 3 \mathrm{~d}$ & ${ }^{2} \mathrm{D}_{3 / 2}^{3 / 2}$ & $-0.57(92)+0.59(15)+0.30(24)+0.37(50)+0.22(44)-0.21(52)$ \\
\hline 45 & $2 \mathrm{~s}^{2} 2 \mathrm{p}^{4}\left({ }^{3} \mathrm{P}\right) 3 \mathrm{~d}$ & ${ }^{4} \mathrm{~F}_{5 / 2}$ & $0.75(45)+0.37(49)+0.44(23)+0.24(51)$ \\
\hline 46 & $2 \mathrm{~s}^{2} 2 \mathrm{p}^{4}\left({ }^{1} \mathrm{D}\right) 3 \mathrm{~d}$ & ${ }^{2} \mathrm{G}_{7 / 2}$ & $0.38(47)+0.39(18)-0.79(46)+0.27(57)$ \\
\hline 47 & $2 \mathrm{~s}^{2} 2 \mathrm{p}^{4}\left({ }^{3} \mathrm{P}\right) 3 \mathrm{~d}$ & ${ }^{4} \mathrm{~F}_{7 / 2}$ & $0.65(47)+0.64(19)-0.37(18)$ \\
\hline 48 & $2 \mathrm{~s}^{2} 2 \mathrm{p}^{4}\left({ }^{1} \mathrm{D}\right) 3 \mathrm{~d}$ & ${ }^{2} \mathrm{~S}_{1 / 2}$ & $-0.56(17)-0.45(60)+0.68(48)$ \\
\hline 49 & $2 \mathrm{~s}^{2} 2 \mathrm{p}^{4}\left({ }^{3} \mathrm{P}\right) 3 \mathrm{~d}$ & ${ }^{2} \mathrm{~F}_{5 / 2}$ & $-0.23(45)+0.42(16)+0.55(93)+0.65(49)$ \\
\hline 50 & $2 \mathrm{~s}^{2} 2 \mathrm{p}^{4}\left({ }^{3} \mathrm{P}\right) 3 \mathrm{~d}$ & ${ }^{2} \mathrm{P}_{3 / 2}$ & $-0.30(92)-0.42(15)-0.22(24)+0.32(58)+0.64(50)-0.39(44)$ \\
\hline 51 & $2 \mathrm{~s}^{2} 2 \mathrm{p}^{4}\left({ }^{1} \mathrm{D}\right) 3 \mathrm{~d}$ & ${ }^{2} \mathrm{~F}_{5 / 2}$ & $0.30(16)-0.34(93)+0.39(23)-0.65(51)+0.40(56)$ \\
\hline 52 & $2 \mathrm{~s}^{2} 2 \mathrm{p}^{4}\left({ }^{1} \mathrm{D}\right) 3 \mathrm{~d}$ & ${ }^{2} \mathrm{P}_{3 / 2}$ & $0.58(24)-0.35(44)+0.70(52)$ \\
\hline 53 & $2 \mathrm{~s}^{2} 2 \mathrm{p}^{4}\left({ }^{1} \mathrm{D}\right) 3 \mathrm{~d}$ & ${ }^{2} \mathrm{G}_{9 / 2}$ & $0.55(20)-0.83(53)$ \\
\hline 54 & $2 \mathrm{~s} 2 \mathrm{p}^{5}\left({ }^{1} \mathrm{P}\right) 3 \mathrm{~s}$ & ${ }^{2} \mathrm{P}_{1 / 2}^{o}$ & $-0.39(88)-0.52(91)+0.74(54)$ \\
\hline 55 & $2 \mathrm{~s} 2 \mathrm{p}^{5}\left({ }^{3} \mathrm{P}\right) 3 \mathrm{~s}$ & ${ }^{4} \mathrm{P}_{3 / 2}^{\mathrm{o}}$ & $-0.53(55)+0.44(43)+0.72(90)$ \\
\hline 56 & $2 \mathrm{~s}^{2} 2 \mathrm{p}^{4}\left({ }^{1} \mathrm{D}\right) 3 \mathrm{~d}$ & ${ }^{2} \mathrm{D}_{5 / 2}$ & $0.33(93)+0.37(23)-0.44(51)-0.71(56)$ \\
\hline 57 & $2 \mathrm{~s}^{2} 2 \mathrm{p}^{4}\left({ }^{1} \mathrm{D}\right) 3 \mathrm{~d}$ & ${ }^{2} \mathrm{~F}_{7 / 2}$ & $-0.29(47)+0.30(19)+0.38(18)-0.26(46)-0.79(57)$ \\
\hline 58 & $2 \mathrm{~s}^{2} 2 \mathrm{p}^{4}\left({ }^{3} \mathrm{P}\right) 3 \mathrm{~d}$ & ${ }^{2} \mathrm{D}_{3 / 2}$ & $0.25(92)-0.56(58)-0.50(44)-0.37(52)+0.30(97)-0.25(59)$ \\
\hline 59 & $2 \mathrm{~s} 2 \mathrm{p}^{5}\left({ }^{3} \mathrm{P}\right) 3 \mathrm{p}$ & ${ }^{4} \mathrm{~S}_{3 / 2}$ & $0.29(58)+0.32(44)-0.29(64)+0.58(97)-0.51(59)+0.27(66)$ \\
\hline
\end{tabular}


Table B (continued)

\begin{tabular}{|c|c|c|c|}
\hline Index & Configuration & Level & Mixing coefficients \\
\hline 60 & $2 \mathrm{~s}^{2} 2 \mathrm{p}^{4}\left({ }^{1} \mathrm{D}\right) 3 \mathrm{~d}$ & ${ }^{2} \mathrm{P}_{1 / 2}$ & $-0.57(21)-0.63(60)-0.45(48)$ \\
\hline 61 & $2 \mathrm{~s} 2 \mathrm{p}^{5}\left({ }^{3} \mathrm{P}\right) 3 \mathrm{p}$ & ${ }^{2} \mathrm{D}_{5 / 2}$ & $0.63(70)-0.40(67)+0.63(61)$ \\
\hline 62 & $2 \mathrm{~s}^{2} 2 \mathrm{p}^{4} 3 \mathrm{~s}$ & ${ }^{4} \mathrm{P}_{1 / 2}$ & $-0.61(62)+0.45(27)+0.59(6)$ \\
\hline 63 & $2 \mathrm{~s} 2 \mathrm{p}^{5}\left({ }^{1} \mathrm{P}\right) 3 \mathrm{p}$ & ${ }^{2} \mathrm{P}_{1 / 2}$ & $0.34(94)-0.47(96)-0.30(98)+0.58(63)-0.46(72)$ \\
\hline 64 & $2 \mathrm{~s} 2 \mathrm{p}^{5}\left({ }^{3} \mathrm{P}\right) 3 \mathrm{p}$ & ${ }^{4} \mathrm{D}_{3 / 2}$ & $-0.52(64)+0.21(59)-0.27(71)-0.26(66)+0.67(95)-0.30(100)$ \\
\hline 65 & $2 \mathrm{~s} 2 \mathrm{p}^{5}\left({ }^{3} \mathrm{P}\right) 3 \mathrm{p}$ & ${ }^{4} \mathrm{D}_{7 / 2}$ & $1.00(65)$ \\
\hline 66 & $2 \mathrm{~s} 2 \mathrm{p}^{5}\left({ }^{3} \mathrm{P}\right) 3 \mathrm{p}$ & ${ }^{2} \mathrm{P}_{3 / 2}$ & $0.51(59)-0.28(71)+0.79(66)$ \\
\hline 67 & $2 \mathrm{~s} 2 \mathrm{p}^{5}\left({ }^{3} \mathrm{P}\right) 3 \mathrm{p}$ & ${ }^{4} \mathrm{P}_{5 / 2}$ & $0.75(67)+0.64(61)$ \\
\hline 68 & $2 \mathrm{~s} 2 \mathrm{p}^{5}\left({ }^{3} \mathrm{P}\right) 3 \mathrm{p}$ & ${ }^{2} \mathrm{P}_{1 / 2}$ & $0.26(94)-0.26(96)-0.66(68)+0.58(98)+0.25(72)$ \\
\hline 69 & $2 \mathrm{~s}^{2} 2 \mathrm{p}^{4}\left({ }^{3} \mathrm{P}\right) 3 \mathrm{p}$ & ${ }^{4} \mathrm{D}_{1 / 2}^{o}$ & $-0.56(69)-0.24(30)-0.43(12)-0.26(35)+0.60(9)$ \\
\hline 70 & $2 \mathrm{~s} 2 \mathrm{p}^{5}\left({ }^{3} \mathrm{P}\right) 3 \mathrm{p}$ & ${ }^{4} \mathrm{D}_{5 / 2}$ & $-0.50(70)-0.35(67)+0.28(61)+0.74(99)$ \\
\hline 71 & $2 \mathrm{~s} 2 \mathrm{p}^{5}\left({ }^{3} \mathrm{P}\right) 3 \mathrm{p}$ & $\begin{array}{l}2 \mathrm{D}_{3 / 2} \\
{ }^{2}\end{array}$ & $-0.36(97)-0.30(59)-0.47(71)+0.30(95)+0.67(100)$ \\
\hline 72 & $2 \mathrm{~s} 2 \mathrm{p}^{5}\left({ }^{1} \mathrm{P}\right) 3 \mathrm{p}$ & ${ }^{2} \mathrm{~S}_{1 / 2}$ & $0.20(94)+0.49(96)-0.20(68)-0.45(98)+0.46(63)+0.51(72)$ \\
\hline 73 & $2 \mathrm{~s} 2 \mathrm{p}^{5}\left({ }^{3} \mathrm{P}\right) 3 \mathrm{~d}$ & ${ }^{4} \mathrm{P}_{1 / 2}^{0}$ & $0.29(105)-0.95(73)$ \\
\hline 74 & $2 \mathrm{~s} 2 \mathrm{p}^{5}\left({ }^{3} \mathrm{P}\right) 3 \mathrm{~d}$ & ${ }^{4} \mathrm{P}_{3 / 2}^{1 / 2}$ & $-0.58(107)+0.78(74)$ \\
\hline 75 & $2 \mathrm{~s} 2 \mathrm{p}^{5}\left({ }^{3} \mathrm{P}\right) 3 \mathrm{~d}$ & ${ }^{4} \mathrm{~F}_{7 / 2}^{3 / 2}$ & $0.72(75)-0.36(80)+0.59(86)$ \\
\hline 76 & $2 \mathrm{~s} 2 \mathrm{p}^{5}\left({ }^{3} \mathrm{P}\right) 3 \mathrm{~d}$ & ${ }^{4} \mathrm{D}_{5 / 2}^{o}$ & $-0.48(102)+0.71(76)-0.38(103)+0.30(79)$ \\
\hline 77 & $2 \mathrm{~s} 2 \mathrm{p}^{5}\left({ }^{3} \mathrm{P}\right) 3 \mathrm{~d}$ & ${ }^{4} \mathrm{~F}_{9 / 2}^{o}$ & $1.00(77)$ \\
\hline 78 & $2 \mathrm{~s}^{2} 2 \mathrm{p}^{4}\left({ }^{1} \mathrm{~S}\right) 3 \mathrm{p}$ & ${ }^{2} \mathrm{P}_{3 / 2}^{o / 2}$ & $-0.41(31)-0.40(7)-0.27(13)+0.42(36)+0.60(78)$ \\
\hline 79 & $2 \mathrm{~s} 2 \mathrm{p}^{5}\left({ }^{3} \mathrm{P}\right) 3 \mathrm{~d}$ & ${ }^{2} \mathrm{D}_{5 / 2}^{o / 2}$ & $-0.26(102)-0.26(76)+0.55(103)-0.37(108)+0.62(79)$ \\
\hline 80 & $2 \mathrm{~s} 2 \mathrm{p}^{5}\left({ }^{3} \mathrm{P}\right) 3 \mathrm{~d}$ & ${ }^{4} \mathrm{D}_{7 / 2}^{o}$ & $0.73(80)+0.66(86)$ \\
\hline 81 & $2 \mathrm{~s} 2 \mathrm{p}^{5}\left({ }^{3} \mathrm{P}\right) 3 \mathrm{~d}$ & ${ }^{2} \mathrm{D}_{3 / 2}^{o}$ & $0.39(101)-0.25(107)-0.38(74)-0.62(81)+0.37(85)+0.33(104)$ \\
\hline 82 & $2 \mathrm{~s} 2 \mathrm{p}^{5}\left({ }^{1} \mathrm{P}\right) 3 \mathrm{~d}$ & ${ }^{2} \mathrm{P}_{1 / 2}^{o}$ & $0.36(105)+0.63(84)+0.69(82)$ \\
\hline 83 & $2 \mathrm{~s} 2 \mathrm{p}^{5}\left({ }^{1} \mathrm{P}\right) 3 \mathrm{~d}$ & ${ }^{2} \mathrm{~F}_{5 / 2}^{o}$ & $-0.38(102)-0.20(108)-0.50(79)+0.72(83)$ \\
\hline 84 & $2 \mathrm{~s} 2 \mathrm{p}^{5}\left({ }^{3} \mathrm{P}\right) 3 \mathrm{~d}$ & ${ }^{2} \mathrm{P}_{1 / 2}^{\circ / 2}$ & $0.62(105)+0.21(73)-0.67(84)+0.29(82)$ \\
\hline 85 & $2 \mathrm{~s} 2 \mathrm{p}^{5}\left({ }^{3} \mathrm{P}\right) 3 \mathrm{~d}$ & ${ }^{2} \mathrm{P}_{3 / 2}^{0}$ & $0.27(101)-0.27(107)+0.37(81)-0.47(85)+0.54(104)-0.43(89)$ \\
\hline 86 & $2 \mathrm{~s} 2 \mathrm{p}^{5}\left({ }^{3} \mathrm{P}\right) 3 \mathrm{~d}$ & ${ }^{2} \mathrm{~F}_{7 / 2}^{o / 2}$ & $-0.42(75)-0.39(80)+0.35(86)+0.74(106)$ \\
\hline 87 & $2 \mathrm{~s} 2 \mathrm{p}^{5}\left({ }^{1} \mathrm{P}\right) 3 \mathrm{~d}$ & ${ }^{2} \mathrm{D}_{5 / 2}^{o}$ & $0.20(102)-0.25(76)-0.37(103)-0.47(108)+0.71(87)$ \\
\hline 88 & $2 \mathrm{~s} 2 \mathrm{p}^{5}\left({ }^{3} \mathrm{P}\right) 3 \mathrm{~s}$ & ${ }^{4} \mathrm{P}_{1 / 2}^{o}$ & $-0.87(88)+0.42(91)$ \\
\hline 89 & $2 \mathrm{~s} 2 \mathrm{p}^{5}\left({ }^{1} \mathrm{P}\right) 3 \mathrm{~d}$ & ${ }^{2} \mathrm{P}_{3 / 2}^{1 / 2}$ & $0.36(107)+0.21(74)-0.23(81)-0.43(85)+0.39(104)+0.59(89)$ \\
\hline 90 & $2 \mathrm{~s} 2 \mathrm{p}^{5}\left({ }^{1} \mathrm{P}\right) 3 \mathrm{~s}$ & ${ }^{2} \mathrm{P}_{3 / 2}^{3 / 2}$ & $0.64(55)-0.30(43)+0.64(90)$ \\
\hline 91 & $2 \mathrm{~s} 2 \mathrm{p}^{5}\left({ }^{3} \mathrm{P}\right) 3 \mathrm{~s}$ & $2 \begin{array}{c}3 / 2 \\
\mathrm{P}_{1 / 2}^{o}\end{array}$ & $0.24(88)+0.73(91)+0.64(54)$ \\
\hline 92 & $2 \mathrm{~s}^{2} 2 \mathrm{p}^{4}\left({ }^{3} \mathrm{P}\right) 3 \mathrm{~d}$ & ${ }^{4} \mathrm{~F}_{3 / 2}$ & $-0.52(92)-0.30(15)-0.36(58)-0.33(50)+0.60(22)$ \\
\hline 93 & $2 \mathrm{~s}^{2} 2 \mathrm{p}^{4}\left({ }^{3} \mathrm{P}\right) 3 \mathrm{~d}$ & ${ }^{4} \mathrm{P}_{5 / 2}$ & $-0.36(45)-0.39(16)-0.36(93)+0.41(49)+0.22(23)+0.61(25)$ \\
\hline 94 & $2 \mathrm{~s} 2 \mathrm{p}^{5}\left({ }^{3} \mathrm{P}\right) 3 \mathrm{p}$ & ${ }^{4} \mathrm{D}_{1 / 2}$ & $-0.81(94)-0.46(68)-0.24(98)$ \\
\hline 95 & $2 \mathrm{~s} 2 \mathrm{p}^{5}\left({ }^{1} \mathrm{P}\right) 3 \mathrm{p}$ & ${ }^{2} \mathrm{D}_{3 / 2}$ & $0.56(64)+0.25(71)+0.37(66)+0.60(95)-0.29(100)$ \\
\hline 96 & $2 \mathrm{~s} 2 \mathrm{p}^{5}\left({ }^{3} \mathrm{P}\right) 3 \mathrm{p}$ & ${ }^{4} \mathrm{P}_{1 / 2}$ & $0.54(96)+0.50(98)+0.49(63)-0.41(72)$ \\
\hline 97 & $2 \mathrm{~s} 2 \mathrm{p}^{5}\left({ }^{3} \mathrm{P}\right) 3 \mathrm{p}$ & ${ }^{4} \mathrm{P}_{3 / 2}$ & $-0.50(64)-0.56(97)-0.36(59)+0.49(71)+0.25(66)$ \\
\hline 98 & $2 \mathrm{~s} 2 \mathrm{p}^{5}\left({ }^{3} \mathrm{P}\right) 3 \mathrm{p}$ & ${ }^{2} \mathrm{~S}_{1 / 2}$ & $-0.27(94)-0.37(96)+0.54(68)+0.22(98)+0.39(63)+0.52(72)$ \\
\hline 99 & $2 \mathrm{~s} 2 \mathrm{p}^{5}\left({ }^{1} \mathrm{P}\right) 3 \mathrm{p}$ & ${ }^{2} \mathrm{D}_{5 / 2}$ & $0.56(70)+0.37(67)-0.31(61)+0.67(99)$ \\
\hline 100 & $2 \mathrm{~s} 2 \mathrm{p}^{5}\left({ }^{1} \mathrm{P}\right) 3 \mathrm{p}$ & ${ }^{2} \mathrm{P}_{3 / 2}$ & $0.33(97)+0.35(59)+0.54(71)+0.28(95)+0.61(100)$ \\
\hline 101 & $2 \mathrm{~s} 2 \mathrm{p}^{5}\left({ }^{3} \mathrm{P}\right) 3 \mathrm{~d}$ & ${ }^{4} \mathrm{~F}_{3 / 2}^{o}$ & $-0.79(101)-0.33(107)-0.37(81)-0.28(85)+0.21(104)$ \\
\hline 102 & $2 \mathrm{~s} 2 \mathrm{p}^{5}\left({ }^{3} \mathrm{P}\right) 3 \mathrm{~d}$ & ${ }^{4} \mathrm{~F}_{5 / 2}^{o}$ & $-0.58(102)-0.56(76)-0.41(103)+0.34(108)-0.24(83)$ \\
\hline 103 & $2 \mathrm{~s} 2 \mathrm{p}^{5}\left({ }^{3} \mathrm{P}\right) 3 \mathrm{~d}$ & ${ }^{4} \mathrm{P}_{5 / 2}^{o}$ & $0.39(102)-0.37(103)+0.31(108)+0.45(79)+0.55(83)-0.33(87)$ \\
\hline 104 & $2 \mathrm{~s} 2 \mathrm{p}^{5}\left({ }^{1} \mathrm{P}\right) 3 \mathrm{~d}$ & ${ }^{2} \mathrm{D}_{3 / 2}^{o / 2}$ & $-0.22(101)+0.47(107)+0.37(74)+0.40(85)+0.50(104)-0.42(89)$ \\
\hline 105 & $2 \mathrm{~s} 2 \mathrm{p}^{5}\left({ }^{3} \mathrm{P}\right) 3 \mathrm{~d}$ & ${ }^{4} \mathrm{D}_{1 / 2}^{3 / 2}$ & $-0.62(105)-0.36(84)+0.65(82)$ \\
\hline 106 & $2 \mathrm{~s} 2 \mathrm{p}^{5}\left({ }^{1} \mathrm{P}\right) 3 \mathrm{~d}$ & ${ }^{2} \mathrm{~F}_{7 / 2}^{o}$ & $0.52(75)+0.43(80)-0.31(86)+0.67(106)$ \\
\hline 107 & $2 \mathrm{~s} 2 \mathrm{p}^{5}\left({ }^{3} \mathrm{P}\right) 3 \mathrm{~d}$ & ${ }^{4} \mathrm{D}_{3 / 2}^{o}$ & $-0.23(107)+0.52(81)+0.44(85)+0.38(104)+0.52(89)$ \\
\hline 108 & $2 \mathrm{~s} 2 \mathrm{p}^{5}\left({ }^{3} \mathrm{P}\right) 3 \mathrm{~d}$ & ${ }^{2} \mathrm{~F}_{5 / 2}^{0}$ & $0.35(103)+0.60(108)+0.29(83)+0.60(87)$ \\
\hline 109 & $2 \mathrm{p}^{6} 3 \mathrm{~s}$ & ${ }^{2} \mathrm{~S}_{1 / 2}^{5 / 2}$ & $0.98(109)$ \\
\hline 110 & $2 p^{6} 3 p$ & ${ }^{2} \mathrm{P}_{1 / 2}^{o}$ & $0.98(110)$ \\
\hline 111 & $2 \mathrm{p}^{6} 3 \mathrm{p}$ & ${ }^{2} \mathrm{P}_{3 / 2}^{o}$ & $0.99(111)$ \\
\hline 112 & $2 \mathrm{p}^{6} 3 \mathrm{~d}$ & ${ }^{2} \mathrm{D}_{3 / 2}^{3 / 2}$ & $1.00(112)$ \\
\hline 113 & $2 \mathrm{p}^{6} 3 \mathrm{~d}$ & ${ }^{2} \mathrm{D}_{5 / 2}$ & $1.00(113)$ \\
\hline
\end{tabular}


Table C

Comparison of energy levels (in Ryd) for F-like Ba XLVIII.

\begin{tabular}{|c|c|c|c|c|c|c|}
\hline S.No. & Configuration & Level & Index & NIST & GRASP & FAC \\
\hline 1 & $2 \mathrm{~s}^{2} 2 \mathrm{p}^{5}$ & ${ }^{2} \mathrm{P}_{3 / 2}^{o}$ & 1 & 0.000 & 0.0000 & 0.0000 \\
\hline 2 & $2 \mathrm{~s}^{2} 2 \mathrm{p}^{5}$ & ${ }^{2} \mathrm{P}_{1 / 2}^{o}$ & 2 & 28.985 & 28.9304 & 28.9524 \\
\hline 3 & $2 \mathrm{~s} 2 \mathrm{p}^{6}$ & ${ }^{2} \mathrm{~S}_{1 / 2}$ & 3 & 52.450 & 52.6383 & 52.6054 \\
\hline 4 & $2 s^{2} 2 p^{4} 3 s$ & ${ }^{4} \mathrm{P}_{5 / 2}$ & 4 & 343.793 & 343.7281 & 343.7235 \\
\hline 5 & $2 s^{2} 2 p^{4} 3 s$ & ${ }^{2} \mathrm{P}_{3 / 2}$ & 5 & 344.366 & 344.3027 & 344.2899 \\
\hline 6 & $2 s^{2} 2 p^{4} 3 s$ & ${ }^{2} \mathrm{~S}_{1 / 2}$ & 6 & 346.930 & 346.8856 & 346.9050 \\
\hline 7 & $2 \mathrm{~s}^{2} 2 \mathrm{p}^{4}\left({ }^{3} \mathrm{P}\right) 3 \mathrm{p}$ & ${ }^{2} \mathrm{D}_{5 / 2}^{o}$ & 8 & 350.487 & 350.5544 & 350.5334 \\
\hline 8 & $2 \mathrm{~s}^{2} 2 \mathrm{p}^{4}\left({ }^{3} \mathrm{P}\right) 3 \mathrm{p}$ & ${ }^{4} \mathrm{~S}_{3 / 2}^{o}$ & 13 & 360.862 & 360.7613 & 360.7373 \\
\hline 9 & $2 \mathrm{~s}^{2} 2 \mathrm{p}^{4}\left({ }^{3} \mathrm{P}\right) 3 \mathrm{~d}$ & ${ }^{2} \mathrm{P}_{1 / 2}$ & 22 & 368.863 & 368.6647 & 368.5919 \\
\hline 10 & $2 \mathrm{~s}^{2} 2 \mathrm{p}^{4}\left({ }^{3} \mathrm{P}\right) 3 \mathrm{~d}$ & ${ }^{2} \mathrm{D}_{5 / 2}$ & 23 & 370.015 & 369.9024 & 369.8235 \\
\hline 11 & $2 \mathrm{~s}^{2} 2 \mathrm{p}^{4}\left({ }^{3} \mathrm{P}\right) 3 \mathrm{~d}$ & ${ }^{2} \mathrm{P}_{3 / 2}$ & 24 & 370.330 & 370.1959 & 370.1377 \\
\hline 12 & $2 \mathrm{~s}^{2} 2 \mathrm{p}^{4}\left({ }^{1} \mathrm{~S}\right) 3 \mathrm{~d}$ & ${ }^{2} \mathrm{D}_{5 / 2}$ & 25 & 371.963 & 371.5784 & 371.5471 \\
\hline 13 & $2 \mathrm{~s}^{2} 2 \mathrm{p}^{4}\left({ }^{1} \mathrm{D}\right) 3 \mathrm{p}$ & ${ }^{2} \mathrm{P}_{3 / 2}^{o}$ & 33 & 382.458 & 382.4383 & 382.3881 \\
\hline 14 & $2 \mathrm{~s} 2 \mathrm{p}^{5}\left({ }^{3} \mathrm{P}\right) 3 \mathrm{~s}$ & ${ }^{2} \mathrm{P}_{3 / 2}^{\mathrm{o}}$ & 43 & 394.380 & 394.4761 & 394.4675 \\
\hline 15 & $2 \mathrm{~s}^{2} 2 \mathrm{p}^{4}\left({ }^{3} \mathrm{P}\right) 3 \mathrm{~d}$ & ${ }^{4} \mathrm{~F}_{5 / 2}$ & 45 & 395.572 & 395.4591 & 395.3894 \\
\hline 16 & $2 \mathrm{~s}^{2} 2 \mathrm{p}^{4}\left({ }^{1} \mathrm{D}\right) 3 \mathrm{~d}$ & ${ }^{2} \mathrm{D}_{3 / 2}$ & 51 & 397.318 & 397.2843 & 397.2045 \\
\hline 17 & $2 \mathrm{~s}^{2} 2 \mathrm{p}^{4}\left({ }^{1} \mathrm{D}\right) 3 \mathrm{~d}$ & ${ }^{2} \mathrm{P}_{3 / 2}$ & 52 & 397.363 & 397.3952 & 397.3195 \\
\hline 18 & $2 \mathrm{~s}^{2} 2 \mathrm{p}^{4}\left({ }^{1} \mathrm{D}\right) 3 \mathrm{~d}$ & ${ }^{2} \mathrm{P}_{1 / 2}$ & 61 & 400.359 & 400.2685 & 400.1764 \\
\hline 19 & $2 \mathrm{~s} 2 \mathrm{p}^{5}\left({ }^{3} \mathrm{P}\right) 3 \mathrm{p}$ & ${ }^{4} \mathrm{P}_{5 / 2}$ & 67 & 408.356 & 408.3451 & 408.3509 \\
\hline
\end{tabular}

Index: see Table 2

NIST: http://www.nist.gov/pml/data/asd.cfm, energies for the lowest 3 levels are extrapolated results from Feldman et al. [11] whereas for the remaining levels are from measurements of Hutton et al. [9]

GRASP: present calculations with the GRASP code for 501 levels

FAC: present calculations with the FAC code for 38089 levels

Table D

Comparison of energies (in Ryd) for the $2 \mathrm{~s}^{2} 2 \mathrm{p}^{5}{ }^{2} \mathrm{P}_{1 / 2}^{o}$ and $2 \mathrm{~s} 2 \mathrm{p}^{6}{ }^{2} \mathrm{~S}_{1 / 2}$ levels of F-like ions with $55 \leq \mathrm{Z} \leq 73$.

\begin{tabular}{|c|c|c|c|c|c|c|c|c|}
\hline \multirow{2}{*}{$\begin{array}{c}\mathrm{Z} \\
\text { Level }\end{array}$} & \multicolumn{2}{|c|}{ Jönsson et al. [13] } & \multicolumn{2}{|c|}{ GRASP } & \multicolumn{2}{|c|}{ FAC } & \multicolumn{2}{|c|}{ MBPT } \\
\hline & ${ }^{2} \mathrm{P}_{1 / 2}^{o}$ & ${ }^{2} \mathrm{~S}_{1 / 2}$ & ${ }^{2} \mathrm{P}_{1 / 2}^{o}$ & ${ }^{2} \mathrm{~S}_{1 / 2}$ & ${ }^{2} \mathrm{P}_{1 / 2}^{o}$ & ${ }^{2} \mathrm{~S}_{1 / 2}$ & ${ }^{2} \mathrm{P}_{1 / 2}^{o}$ & ${ }^{2} \mathrm{~S}_{1 / 2}$ \\
\hline 55 & 26.7247 & 49.6325 & 26.6976 & 49.8336 & 26.7173 & 49.8034 & 26.7550 & 49.6744 \\
\hline 56 & 28.9589 & 52.4309 & 28.9304 & 52.6383 & 28.9524 & 52.6054 & 28.9923 & 52.4764 \\
\hline 57 & 31.3379 & 55.3814 & 31.3081 & 55.5955 & 31.3325 & 55.5597 & 31.3747 & 55.4309 \\
\hline 58 & 33.8689 & 58.4913 & 33.8377 & 58.7126 & 33.8648 & 58.6735 & 33.9093 & 58.5451 \\
\hline 59 & 36.5593 & 61.7680 & 36.5267 & 61.9972 & 36.5566 & 61.9544 & 36.6037 & 61.8263 \\
\hline 60 & 39.4168 & 65.1892 & 39.3827 & 65.4574 & 39.4158 & 65.4098 & 39.4653 & 65.2821 \\
\hline 61 & 42.4496 & 68.8538 & 42.4138 & 69.1014 & 42.4502 & 69.0482 & & \\
\hline 62 & 45.6657 & 72.6734 & 45.6283 & 72.9380 & 45.6682 & 72.8783 & & \\
\hline 63 & 49.0725 & 76.7044 & 49.0348 & 76.9760 & 49.0786 & 76.9091 & & \\
\hline 64 & 52.6814 & 80.9393 & 52.6424 & 81.2249 & 52.6903 & 81.1488 & & \\
\hline 65 & 56.5008 & 85.3943 & 56.4605 & 85.6944 & 56.5127 & 85.6087 & & \\
\hline 66 & 60.5404 & 90.0782 & 60.4987 & 90.3948 & 60.5557 & 90.2975 & & \\
\hline 67 & 64.8103 & 95.0017 & 64.7674 & 95.3365 & 64.8293 & 95.2259 & & \\
\hline 68 & 69.3212 & 100.1762 & 69.2771 & 100.5306 & 69.3443 & 100.4053 & & \\
\hline 69 & 74.0568 & 105.6125 & 74.0389 & 105.9885 & 74.1117 & 105.8459 & & \\
\hline 70 & 79.1104 & 111.3212 & 79.0641 & 111.7224 & 79.1431 & 111.5597 & & \\
\hline 71 & 84.4122 & 117.3168 & 84.3650 & 117.7446 & 84.4505 & 117.5599 & & \\
\hline 72 & 90.0019 & 123.6104 & 89.9541 & 124.0681 & 90.0464 & 123.8577 & & \\
\hline 73 & 95.8926 & 130.2154 & 95.8444 & 130.7068 & 95.9439 & 130.4670 & & \\
\hline
\end{tabular}

GRASP: present calculations with the GRASP code for 501 levels FAC: present calculations with the FAC code for 38089 levels MBPT: earlier calculations of $\mathrm{Gu}[12]$ with the MBPT code 
Table E

Comparison of A-values $\left(\mathrm{s}^{-1}\right)$ for transitions among the lowest three levels of F-like ions with $55 \leq \mathrm{Z} \leq 73$. The first entry is from present calculations with GRASP and the second is from Jönsson et al. [13]. $a \pm b \equiv a \times 10^{ \pm b}$

\begin{tabular}{|c|c|c|c|c|}
\hline $\mathrm{Z}$ & $1-3$ (E1) & $2-3(\mathrm{E} 1)$ & $1-2$ (M1) & $1-2(\mathrm{E} 2)$ \\
\hline 55 & $2.064+12$ & $9.531+10$ & $4.418+08$ & $1.112+06$ \\
\hline 55 & $2.017+12$ & $9.149+10$ & $4.433+08$ & $1.109+06$ \\
\hline 56 & $2.337+12$ & $9.821+10$ & $5.616+08$ & $1.526+06$ \\
\hline 56 & $2.285+12$ & $9.427+10$ & $5.634+08$ & $1.522+06$ \\
\hline 57 & $2.646+12$ & $1.012+11$ & $7.109+08$ & $2.084+06$ \\
\hline 57 & $2.589+12$ & $9.711+10$ & $7.132+08$ & $2.079+06$ \\
\hline 58 & $2.998+12$ & $1.042+11$ & $8.965+08$ & $2.832+06$ \\
\hline 58 & $2.935+12$ & $1.000+11$ & $8.992+08$ & $2.824+06$ \\
\hline 59 & $3.397+12$ & $1.073+11$ & $1.126+09$ & $3.828+06$ \\
\hline 59 & $3.327+12$ & $1.030+11$ & $1.130+09$ & $3.818+06$ \\
\hline 60 & $3.851+12$ & $1.105+11$ & $1.410+09$ & $5.151+06$ \\
\hline 60 & $3.772+12$ & $1.060+11$ & $1.414+09$ & $5.137+06$ \\
\hline 61 & $4.365+12$ & $1.138+11$ & $1.759+09$ & $6.899+06$ \\
\hline 61 & $4.278+12$ & $1.091+11$ & $1.764+09$ & $6.884+06$ \\
\hline 62 & $4.948+12$ & $1.171+11$ & $2.187+09$ & $9.199+06$ \\
\hline 62 & $4.850+12$ & $1.122+11$ & $2.193+09$ & $9.180+06$ \\
\hline 63 & $5.610+12$ & $1.205+11$ & $2.710+09$ & $1.221+07$ \\
\hline 63 & $5.503+12$ & $1.155+11$ & $2.717+09$ & $1.217+07$ \\
\hline 64 & $6.360+12$ & $1.240+11$ & $3.348+09$ & $1.615+07$ \\
\hline 64 & $6.240+12$ & $1.188+11$ & $3.357+09$ & $1.610+07$ \\
\hline 65 & $7.210+12$ & $1.276+11$ & $4.124+09$ & $2.127+07$ \\
\hline 65 & $7.075+12$ & $1.221+11$ & $4.135+09$ & $2.121+07$ \\
\hline 66 & $8.173+12$ & $1.313+11$ & $5.066+09$ & $2.792+07$ \\
\hline 66 & $8.022+12$ & $1.255+11$ & $5.079+09$ & $2.783+07$ \\
\hline 67 & $9.264+12$ & $1.351+11$ & $6.206=09$ & $3.650+07$ \\
\hline 67 & $9.093+12$ & $1.290+11$ & $6.221+09$ & $3.638+07$ \\
\hline 68 & $1.050+13$ & $1.390+11$ & $7.582+09$ & $4.755+07$ \\
\hline 68 & $1.031+13$ & $1.326+11$ & $7.600+09$ & $4.739+07$ \\
\hline 69 & $1.190+13$ & $1.430+11$ & $9.239+09$ & $6.173+07$ \\
\hline 69 & $1.168+13$ & $1.363+11$ & $9.260+09$ & $6.153+07$ \\
\hline 70 & $1.348+13$ & $1.471+11$ & $1.123+10$ & $7.988+07$ \\
\hline 70 & $1.323+13$ & $1.400+11$ & $1.126+10$ & $7.962+07$ \\
\hline 71 & $1.527+13$ & $1.514+11$ & $1.362+10$ & $1.031+08$ \\
\hline 71 & $1.499+13$ & $1.438+11$ & $1.365+10$ & $1.027+08$ \\
\hline 72 & $1.729+13$ & $1.557+11$ & $1.648+10$ & $1.325+08$ \\
\hline 72 & $1.698+13$ & $1.477+11$ & $1.651+10$ & $1.321+08$ \\
\hline 73 & $1.958+13$ & $1.602+11$ & $1.989+10$ & $1.699+08$ \\
\hline 73 & $1.923+13$ & $1.517+11$ & $1.993+10$ & $1.694+08$ \\
\hline
\end{tabular}




\section{Explanation of Tables}

Table 1. Energies (Ryd) for 113 levels of Cs XLVII and their lifetimes ( $\tau$, s). For the ground level the energy is absolute whereas for others are comparative.

$\begin{array}{ll}\text { Index } & \text { Level Index } \\ \text { Configuration } & \text { The configuration to which the level belongs } \\ \text { Level } & \text { The } L S J \text { designation of the level } \\ \text { GRASP } & \text { Present energies from the GRASP code with } 38 \text { configurations and } 501 \text { level calculations } \\ \text { FAC } & \text { Present energies from the FAC code with } 38089 \text { level calculations } \\ \tau(\mathrm{s}) & \text { Lifetime of the level in s with the GRASP code }\end{array}$

Table 2. Energies (Ryd) for 113 levels of Ba XLVIII and their lifetimes ( $\tau, \mathrm{s})$. For the ground level the energy is absolute whereas for others are comparative.

$\begin{array}{ll}\text { Index } & \text { Level Index } \\ \text { Configuration } & \text { The configuration to which the level belongs } \\ \text { Level } & \text { The } L S J \text { designation of the level } \\ \text { NIST } & \text { Energies compiled by NIST and available at the website http://www.nist.gov/pml/data/asd.cfm } \\ \text { GRASP } & \text { Present energies from the GRASP code with } 38 \text { configurations and } 501 \text { level calculations } \\ \text { FAC1 } & \text { Present energies from the FAC code with } 501 \text { level calculations } \\ \text { FAC2 } & \text { Present energies from the FAC code with } 38089 \text { level calculations } \\ \tau(\mathrm{s}) & \text { Lifetime of the level in s }\end{array}$

Table 3. Energies (Ryd) for 113 levels of La XLIX and their lifetimes ( $\tau$, s). For the ground level the energy is absolute whereas for others are comparative.

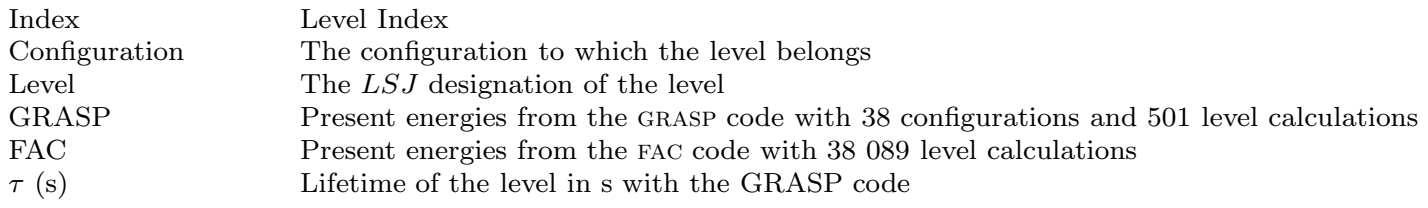

Table 4. Energies (Ryd) for 113 levels of Ce L and their lifetimes ( $\tau, \mathrm{s})$. For the ground level the energy is absolute whereas for others are comparative.

$\begin{array}{ll}\text { Index } & \text { Level Index } \\ \text { Configuration } & \text { The configuration to which the level belongs } \\ \text { Level } & \text { The } L S J \text { designation of the level } \\ \text { GRASP } & \text { Present energies from the GRASP code with } 38 \text { configurations and } 501 \text { level calculations } \\ \text { FAC } & \text { Present energies from the FAC code with } 38089 \text { level calculations } \\ \tau(\mathrm{s}) & \text { Lifetime of the level in s with the GRASP code }\end{array}$

Table 5. Energies (Ryd) for 113 levels of Pr LI and their lifetimes $(\tau, \mathrm{s})$. For the ground level the energy is absolute whereas for others are comparative.

$\begin{array}{ll}\text { Index } & \text { Level Index } \\ \text { Configuration } & \text { The configuration to which the level belongs } \\ \text { Level } & \text { The } L S J \text { designation of the level } \\ \text { GRASP } & \text { Present energies from the GRASP code with } 38 \text { configurations and } 501 \text { level calculations } \\ \text { FAC } & \text { Present energies from the FAC code with } 38089 \text { level calculations } \\ \tau(\mathrm{s}) & \text { Lifetime of the level in s with the GRASP code }\end{array}$


Table 6. Energies (Ryd) for 113 levels of Nd LII and their lifetimes ( $\tau$, s). For the ground level the energy is absolute whereas for others are comparative.

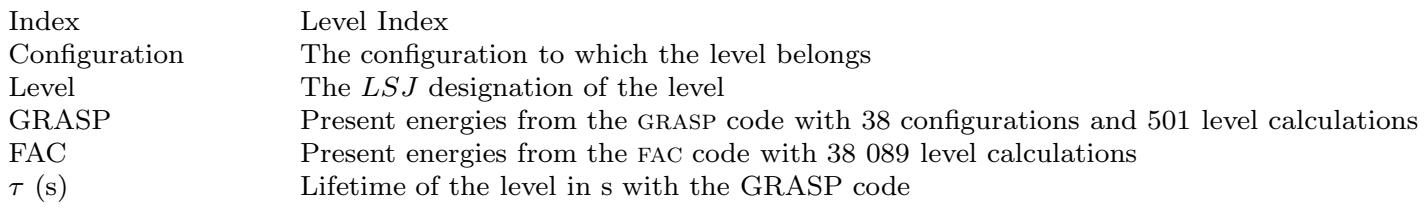

Table 7. Energies (Ryd) for 113 levels of Pm LIII and their lifetimes ( $\tau$, s). For the ground level the energy is absolute whereas for others are comparative.

$\begin{array}{ll}\text { Index } & \text { Level Index } \\ \text { Configuration } & \text { The configuration to which the level belongs } \\ \text { Level } & \text { The } L S J \text { designation of the level } \\ \text { GRASP } & \text { Present energies from the GRASP code with } 38 \text { configurations and } 501 \text { level calculations } \\ \text { FAC } & \text { Present energies from the FAC code with } 38089 \text { level calculations } \\ \tau(\mathrm{s}) & \text { Lifetime of the level in s with the GRASP code }\end{array}$

Table 8. Energies (Ryd) for 113 levels of Sm LIV and their lifetimes ( $\tau$, s). For the ground level the energy is absolute whereas for others are comparative.

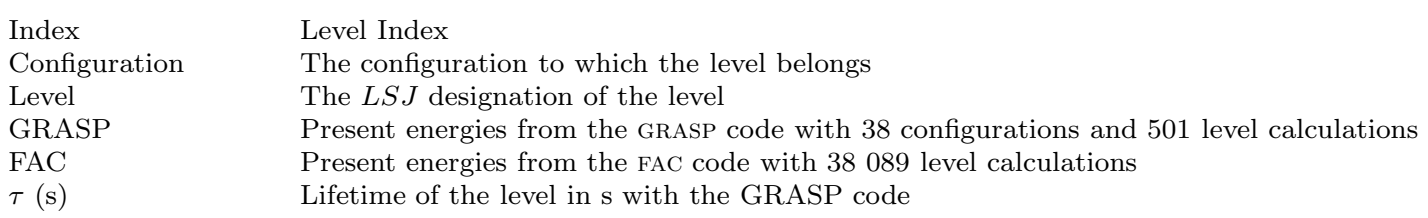

Table 9. Energies (Ryd) for 113 levels of Eu LV and their lifetimes $(\tau$, s). For the ground level the energy is absolute whereas for others are comparative.

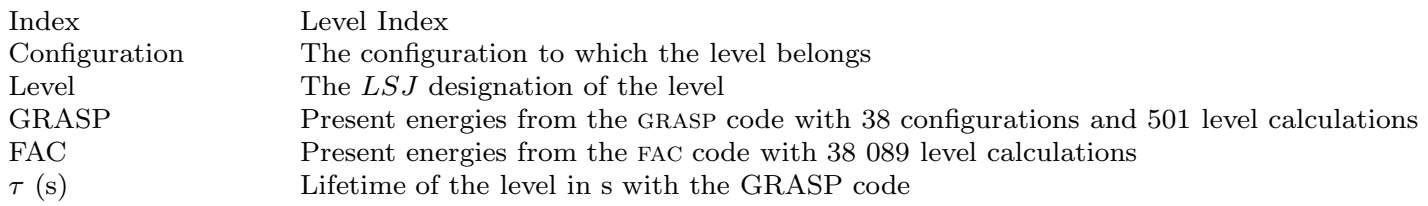

Table 10. Energies (Ryd) for 113 levels of Gd LVI and their lifetimes ( $\tau$, s). For the ground level the energy is absolute whereas for others are comparative.

$\begin{array}{ll}\text { Index } & \text { Level Index } \\ \text { Configuration } & \text { The configuration to which the level belongs } \\ \text { Level } & \text { The } L S J \text { designation of the level } \\ \text { GRASP } & \text { Present energies from the GRASP code with } 38 \text { configurations and } 501 \text { level calculations } \\ \text { FAC } & \text { Present energies from the FAC code with } 38089 \text { level calculations } \\ \tau(\mathrm{s}) & \text { Lifetime of the level in s with the GRASP code }\end{array}$

Table 11. Energies (Ryd) for 113 levels of Tb LVII and their lifetimes ( $\tau$, s). For the ground level the energy is absolute whereas for others are comparative.

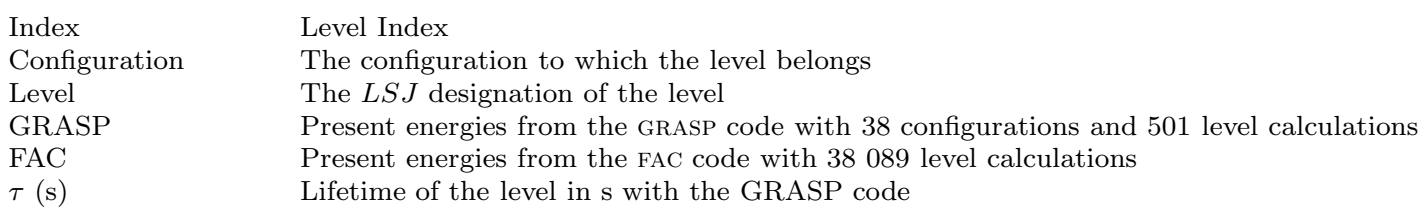


Table 12. Energies (Ryd) for 113 levels of Dy LVIII and their lifetimes $(\tau, \mathrm{s})$. For the ground level the energy is absolute whereas for others are comparative.

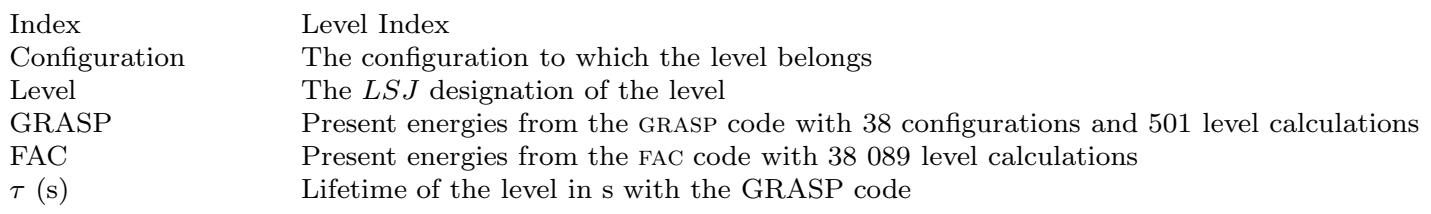

Table 13. Energies (Ryd) for 113 levels of Ho LIX and their lifetimes $(\tau, \mathrm{s})$. For the ground level the energy is absolute whereas for others are comparative.

$\begin{array}{ll}\text { Index } & \text { Level Index } \\ \text { Configuration } & \text { The configuration to which the level belongs } \\ \text { Level } & \text { The } L S J \text { designation of the level } \\ \text { GRASP } & \text { Present energies from the GRASP code with } 38 \text { configurations and } 501 \text { level calculations } \\ \text { FAC } & \text { Present energies from the FAC code with } 38089 \text { level calculations } \\ \tau(\mathrm{s}) & \text { Lifetime of the level in s with the GRASP code }\end{array}$

Table 14. Energies (Ryd) for 113 levels of Er LX and their lifetimes $(\tau, \mathrm{s})$. For the ground level the energy is absolute whereas for others are comparative.

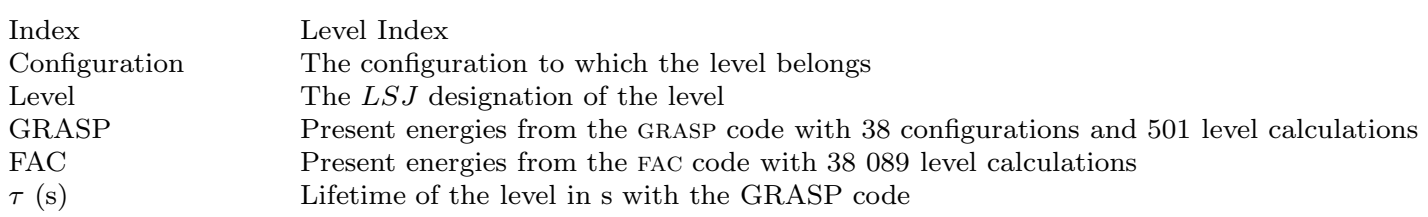

Table 15. Energies (Ryd) for 113 levels of Tm LXI and their lifetimes $(\tau, \mathrm{s})$. For the ground level the energy is absolute whereas for others are comparative.

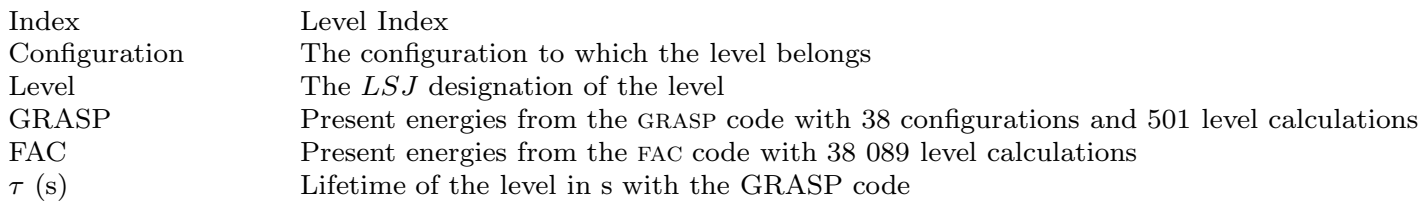

Table 16. Energies (Ryd) for 113 levels of Yb LXII and their lifetimes $(\tau, \mathrm{s})$. For the ground level the energy is absolute whereas for others are comparative.

$\begin{array}{ll}\text { Index } & \text { Level Index } \\ \text { Configuration } & \text { The configuration to which the level belongs } \\ \text { Level } & \text { The LSJ designation of the level } \\ \text { GRASP } & \text { Present energies from the GRASP code with } 38 \text { configurations and } 501 \text { level calculations } \\ \text { FAC } & \text { Present energies from the FAC code with } 38089 \text { level calculations } \\ \tau(\mathrm{s}) & \text { Lifetime of the level in s with the GRASP code }\end{array}$

Table 17. Energies (Ryd) for 113 levels of Lu LXIII and their lifetimes $(\tau, \mathrm{s})$. For the ground level the energy is absolute whereas for others are comparative.

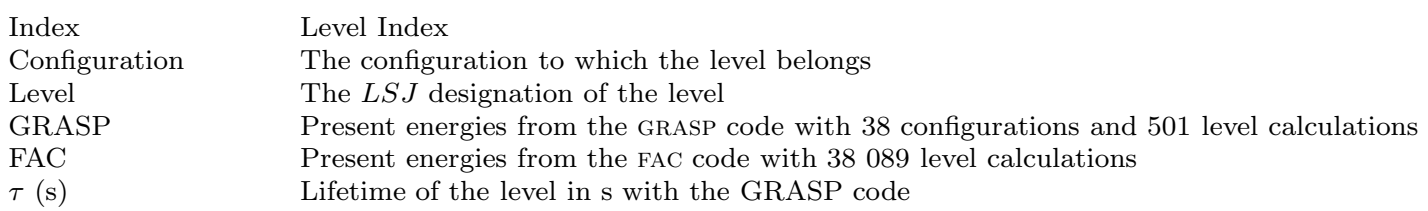


Table 18. Energies (Ryd) for 113 levels of Hf LXIV and their lifetimes $(\tau, \mathrm{s})$. For the ground level the energy is absolute whereas for others are comparative.

$\begin{array}{ll}\text { Index } & \text { Level Index } \\ \text { Configuration } & \text { The configuration to which the level belongs } \\ \text { Level } & \text { The } L S J \text { designation of the level } \\ \text { GRASP } & \text { Present energies from the GRASP code with } 38 \text { configurations and } 501 \text { level calculations } \\ \text { FAC } & \text { Present energies from the FAC code with } 38089 \text { level calculations } \\ \tau(\mathrm{s}) & \text { Lifetime of the level in s with the GRASP code }\end{array}$

Table 19. Energies (Ryd) for 113 levels of Ta LXV and their lifetimes $(\tau$, s). For the ground level the energy is absolute whereas for others are comparative.

$\begin{array}{ll}\text { Index } & \text { Level Index } \\ \text { Configuration } & \text { The configuration to which the level belongs } \\ \text { Level } & \text { The } L S J \text { designation of the level } \\ \text { GRASP } & \text { Present energies from the GRASP code with } 38 \text { configurations and } 501 \text { level calculations } \\ \text { FAC } & \text { Present energies from the FAC code with } 38089 \text { level calculations } \\ \tau(\mathrm{s}) & \text { Lifetime of the level in s with the GRASP code }\end{array}$

Table 20. Transition wavelengths $\left(\lambda_{i j}\right.$ in $\AA$ ), radiative rates $\left(\mathbf{A}_{j i}\right.$ in s $\left.{ }^{-1}\right)$, oscillator strengths $\left(f_{i j}\right.$, dimensionless), and line strengths ( $\mathrm{S}$, in atomic units) for electric dipole (E1), and $\mathrm{A}_{j i}$ for electric quadrupole (E2), magnetic dipole (M1), and magnetic quadrupole (M2) transitions of Cs XLVII. The ratio R(E1) of velocity and length forms of A-values for E1 transitions is listed in the last column.

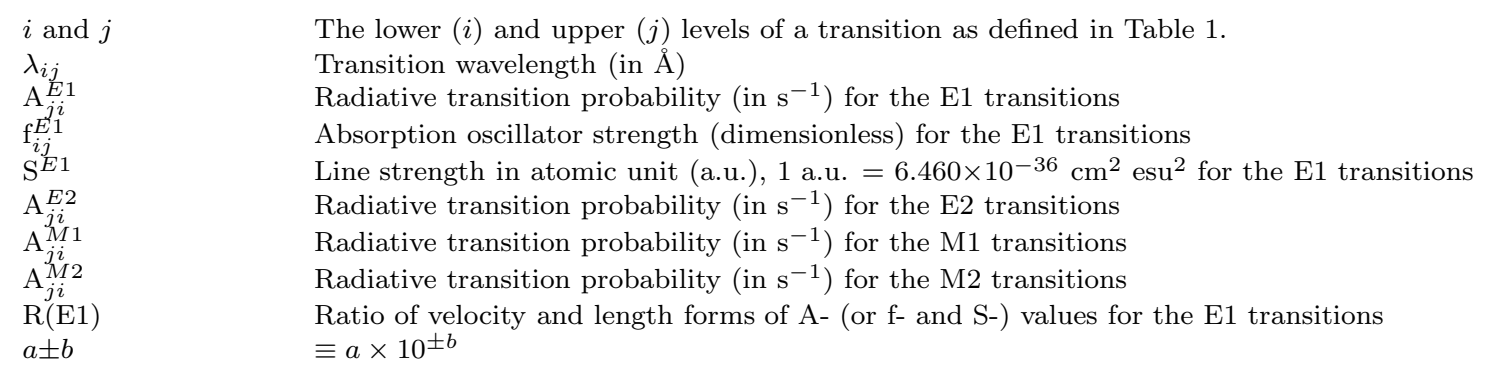

Table 21. Transition wavelengths $\left(\lambda_{i j}\right.$ in $\AA$ ), radiative rates $\left(\mathrm{A}_{j i}\right.$ in $\left.\mathrm{s}^{-1}\right)$, oscillator strengths $\left(\mathrm{f}_{i j}\right.$, dimensionless), and line strengths ( $\mathrm{S}$, in atomic units) for electric dipole (E1), and $\mathbf{A}_{j i}$ for electric quadrupole (E2), magnetic dipole (M1), and magnetic quadrupole (M2) transitions of Ba XLVIII. The ratio R(E1) of velocity and length forms of A-values for E1 transitions is listed in the last column.

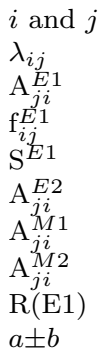

\footnotetext{
The lower $(i)$ and upper $(j)$ levels of a transition as defined in Table 2.

Transition wavelength (in $\AA$ )

Radiative transition probability (in $\mathrm{s}^{-1}$ ) for the E1 transitions

Absorption oscillator strength (dimensionless) for the E1 transitions

Line strength in atomic unit (a.u.), 1 a.u. $=6.460 \times 10^{-36} \mathrm{~cm}^{2} \mathrm{esu}^{2}$ for the E1 transitions

Radiative transition probability (in $\mathrm{s}^{-1}$ ) for the $\mathrm{E} 2$ transitions

Radiative transition probability (in $\mathrm{s}^{-1}$ ) for the M1 transitions

Radiative transition probability (in $\mathrm{s}^{-1}$ ) for the M2 transitions

Ratio of velocity and length forms of A- (or f- and S-) values for the E1 transitions

$\equiv a \times 10^{ \pm b}$
} 
Table 22. Transition wavelengths $\left(\lambda_{i j}\right.$ in $\AA$ ), radiative rates $\left(\mathrm{A}_{j i}\right.$ in $\left.\mathrm{s}^{-1}\right)$, oscillator strengths $\left(\mathrm{f}_{i j}\right.$, dimensionless), and line strengths ( $\mathrm{S}$, in atomic units) for electric dipole (E1), and $\mathrm{A}_{j i}$ for electric quadrupole (E2), magnetic dipole (M1), and magnetic quadrupole (M2) transitions of La XLIX. The ratio R(E1) of velocity and length forms of A-values for E1 transitions is listed in the last column.

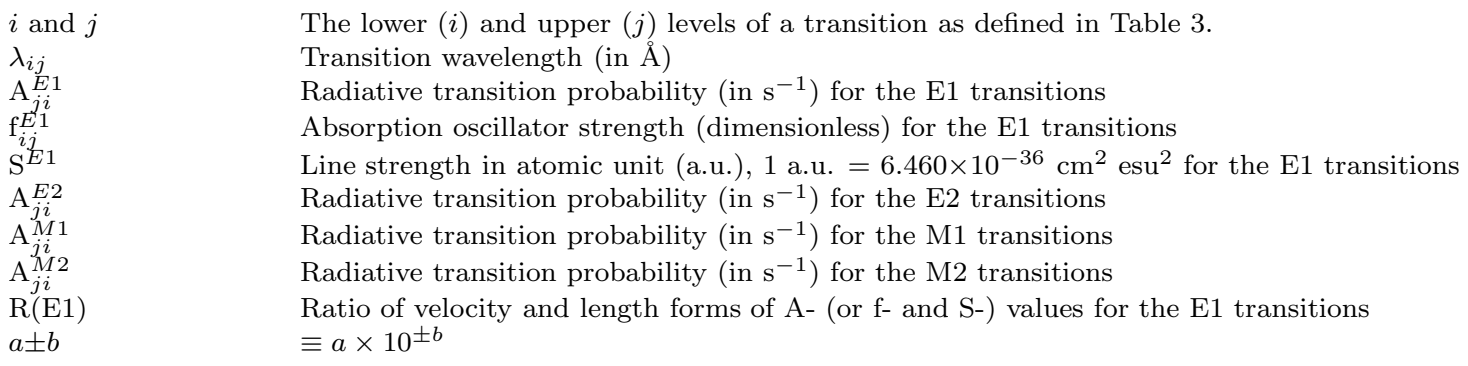

Table 23. Transition wavelengths $\left(\lambda_{i j}\right.$ in $\AA$ ), radiative rates $\left(\mathrm{A}_{j i}\right.$ in $\left.\mathrm{s}^{-1}\right)$, oscillator strengths $\left(\mathrm{f}_{i j}\right.$, dimensionless), and line strengths ( $\mathrm{S}$, in atomic units) for electric dipole (E1), and $\mathrm{A}_{j i}$ for electric quadrupole (E2), magnetic dipole (M1), and magnetic quadrupole (M2) transitions of Ce L. The ratio R(E1) of velocity and length forms of $\mathrm{A}$-values for $\mathrm{E} 1$ transitions is listed in the last column.
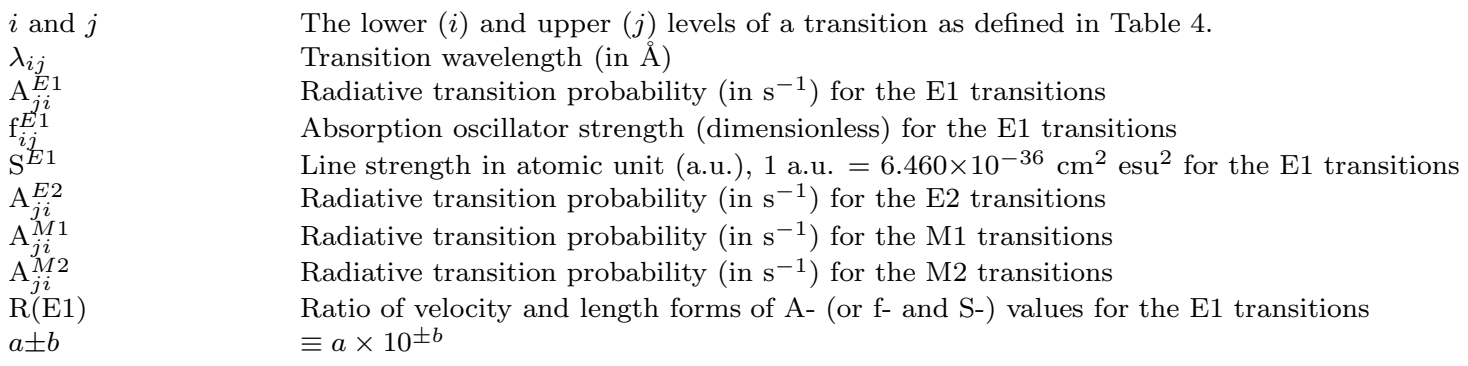

Table 24. Transition wavelengths $\left(\lambda_{i j}\right.$ in $\AA$ ), radiative rates $\left(\mathrm{A}_{j i}\right.$ in $\left.\mathrm{s}^{-1}\right)$, oscillator strengths ( $f_{i j}$, dimensionless), and line strengths ( $\mathrm{S}$, in atomic units) for electric dipole (E1), and $\mathrm{A}_{j i}$ for electric quadrupole (E2), magnetic dipole (M1), and magnetic quadrupole (M2) transitions of Pr LI. The ratio R(E1) of velocity and length forms of A-values for E1 transitions is listed in the last column.

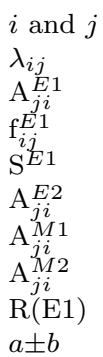

The lower $(i)$ and upper $(j)$ levels of a transition as defined in Table 5 .

Transition wavelength (in $\AA$ )

Radiative transition probability (in $\mathrm{s}^{-1}$ ) for the E1 transitions

Absorption oscillator strength (dimensionless) for the E1 transitions

Line strength in atomic unit (a.u.), 1 a.u. $=6.460 \times 10^{-36} \mathrm{~cm}^{2} \mathrm{esu}^{2}$ for the E1 transitions

Radiative transition probability (in $\mathrm{s}^{-1}$ ) for the E2 transitions

Radiative transition probability (in $\mathrm{s}^{-1}$ ) for the M1 transitions

Radiative transition probability (in $\mathrm{s}^{-1}$ ) for the M2 transitions

Ratio of velocity and length forms of A- (or f- and S-) values for the E1 transitions

$\equiv a \times 10^{ \pm b}$ 
Table 25. Transition wavelengths $\left(\lambda_{i j}\right.$ in $\AA$ ), radiative rates $\left(\mathrm{A}_{j i}\right.$ in $\left.\mathrm{s}^{-1}\right)$, oscillator strengths $\left(\mathrm{f}_{i j}\right.$, dimensionless), and line strengths ( $\mathrm{S}$, in atomic units) for electric dipole (E1), and $\mathbf{A}_{j i}$ for electric quadrupole (E2), magnetic dipole (M1), and magnetic quadrupole (M2) transitions of Nd LII. The ratio R(E1) of velocity and length forms of A-values for E1 transitions is listed in the last column.

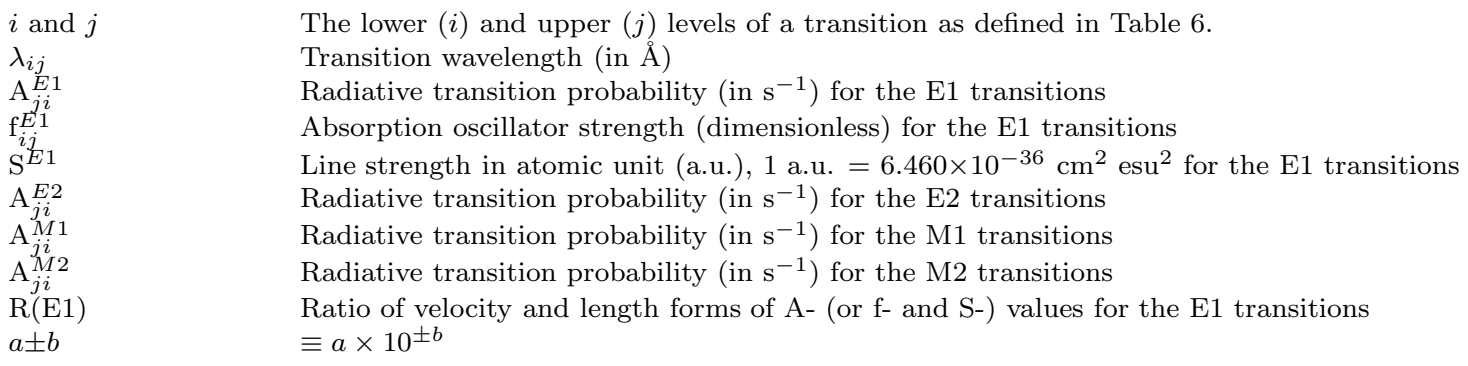

Table 26. Transition wavelengths $\left(\lambda_{i j}\right.$ in $\AA$ ), radiative rates $\left(\mathrm{A}_{j i}\right.$ in $\left.\mathrm{s}^{-1}\right)$, oscillator strengths $\left(\mathrm{f}_{i j}\right.$, dimensionless), and line strengths ( $\mathrm{S}$, in atomic units) for electric dipole (E1), and $\mathrm{A}_{j i}$ for electric quadrupole (E2), magnetic dipole (M1), and magnetic quadrupole (M2) transitions of Pm LIII. The ratio R(E1) of velocity and length forms of $\mathrm{A}$-values for $\mathrm{E} 1$ transitions is listed in the last column.
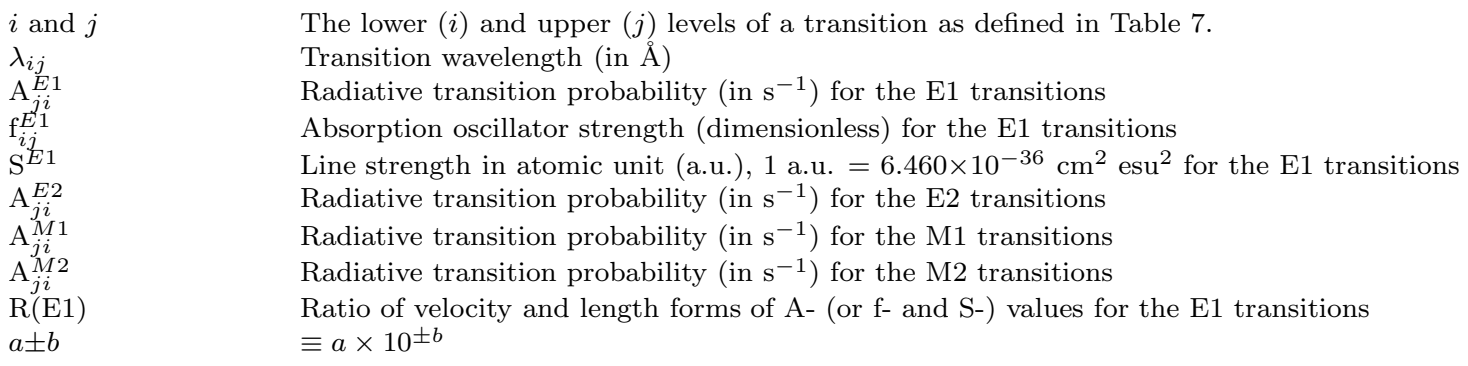

Table 27. Transition wavelengths $\left(\lambda_{i j}\right.$ in $\AA$ ), radiative rates $\left(\mathrm{A}_{j i}\right.$ in $\left.\mathrm{s}^{-1}\right)$, oscillator strengths $\left(\mathrm{f}_{i j}\right.$, dimensionless), and line strengths ( $\mathrm{S}$, in atomic units) for electric dipole (E1), and $\mathrm{A}_{j i}$ for electric quadrupole (E2), magnetic dipole (M1), and magnetic quadrupole (M2) transitions of Sm LIV. The ratio R(E1) of velocity and length forms of A-values for E1 transitions is listed in the last column.

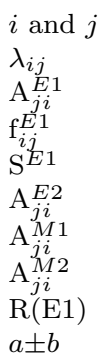

The lower $(i)$ and upper $(j)$ levels of a transition as defined in Table 8.

Transition wavelength (in $\AA$ )

Radiative transition probability (in $\mathrm{s}^{-1}$ ) for the E1 transitions

Absorption oscillator strength (dimensionless) for the E1 transitions

Line strength in atomic unit (a.u.), 1 a.u. $=6.460 \times 10^{-36} \mathrm{~cm}^{2} \mathrm{esu}^{2}$ for the E1 transitions

Radiative transition probability (in $\mathrm{s}^{-1}$ ) for the E2 transitions

Radiative transition probability (in $\mathrm{s}^{-1}$ ) for the M1 transitions

Radiative transition probability (in $\mathrm{s}^{-1}$ ) for the M2 transitions

Ratio of velocity and length forms of A- (or f- and S-) values for the E1 transitions

$\equiv a \times 10^{ \pm b}$ 
Table 28. Transition wavelengths $\left(\lambda_{i j}\right.$ in $\AA$ ), radiative rates $\left(\mathrm{A}_{j i}\right.$ in $\left.\mathrm{s}^{-1}\right)$, oscillator strengths $\left(\mathrm{f}_{i j}\right.$, dimensionless), and line strengths ( $\mathrm{S}$, in atomic units) for electric dipole (E1), and $\mathrm{A}_{j i}$ for electric quadrupole (E2), magnetic dipole (M1), and magnetic quadrupole (M2) transitions of Eu LV. The ratio R(E1) of velocity and length forms of A-values for E1 transitions is listed in the last column.

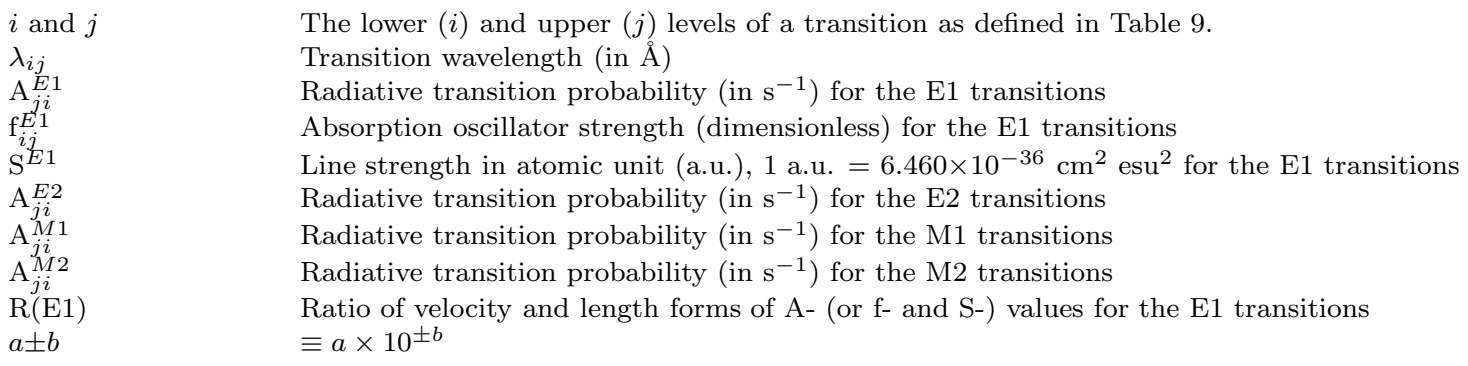

Table 29. Transition wavelengths $\left(\lambda_{i j}\right.$ in $\AA$ ), radiative rates $\left(\mathrm{A}_{j i}\right.$ in $\left.\mathrm{s}^{-1}\right)$, oscillator strengths $\left(\mathrm{f}_{i j}\right.$, dimensionless), and line strengths ( $\mathrm{S}$, in atomic units) for electric dipole (E1), and $\mathrm{A}_{j i}$ for electric quadrupole (E2), magnetic dipole (M1), and magnetic quadrupole (M2) transitions of Gd LVI. The ratio R(E1) of velocity and length forms of A-values for E1 transitions is listed in the last column.
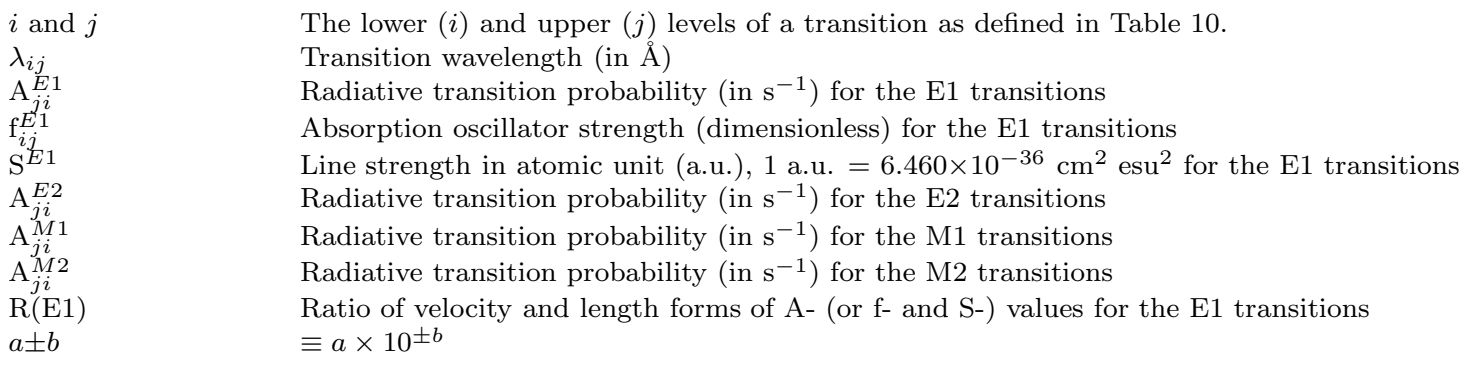

Table 30. Transition wavelengths $\left(\lambda_{i j}\right.$ in $\AA$ ) , radiative rates $\left(\mathrm{A}_{j i}\right.$ in $\left.\mathrm{s}^{-1}\right)$, oscillator strengths $\left(\mathrm{f}_{i j}\right.$, dimensionless), and line strengths ( $\mathrm{S}$, in atomic units) for electric dipole (E1), and $\mathrm{A}_{j i}$ for electric quadrupole (E2), magnetic dipole (M1), and magnetic quadrupole (M2) transitions of Tb LVII. The ratio R(E1) of velocity and length forms of A-values for E1 transitions is listed in the last column.

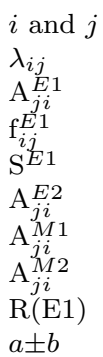

The lower $(i)$ and upper $(j)$ levels of a transition as defined in Table 11.

Transition wavelength (in $\AA$ )

Radiative transition probability (in $\mathrm{s}^{-1}$ ) for the E1 transitions

Absorption oscillator strength (dimensionless) for the E1 transitions

Line strength in atomic unit (a.u.), 1 a.u. $=6.460 \times 10^{-36} \mathrm{~cm}^{2} \mathrm{esu}^{2}$ for the E1 transitions

Radiative transition probability (in $\mathrm{s}^{-1}$ ) for the E2 transitions

Radiative transition probability (in $\mathrm{s}^{-1}$ ) for the M1 transitions

Radiative transition probability (in $\mathrm{s}^{-1}$ ) for the M2 transitions

Ratio of velocity and length forms of A- (or f- and S-) values for the E1 transitions

$\equiv a \times 10^{ \pm b}$ 
Table 31. Transition wavelengths $\left(\lambda_{i j}\right.$ in $\left.\AA\right)$, radiative rates $\left(\mathrm{A}_{j i}\right.$ in $\left.\mathrm{s}^{-1}\right)$, oscillator strengths $\left(\mathrm{f}_{i j}\right.$, dimensionless), and line strengths ( $\mathrm{S}$, in atomic units) for electric dipole (E1), and $\mathbf{A}_{j i}$ for electric quadrupole (E2), magnetic dipole (M1), and magnetic quadrupole (M2) transitions of Dy LVIII. The ratio R(E1) of velocity and length forms of A-values for E1 transitions is listed in the last column.

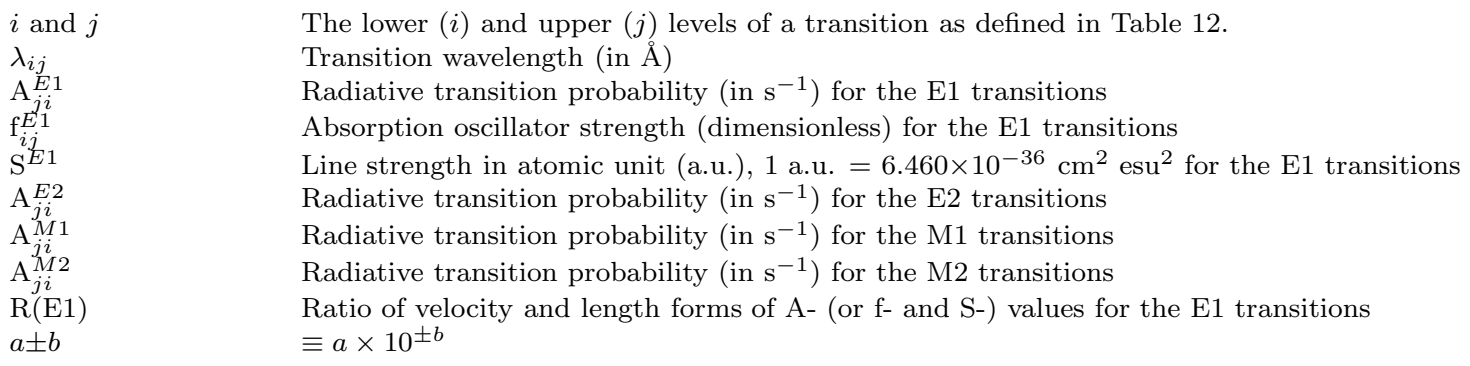

Table 32. Transition wavelengths $\left(\lambda_{i j}\right.$ in $\AA$ ), radiative rates $\left(\mathrm{A}_{j i}\right.$ in $\left.\mathrm{s}^{-1}\right)$, oscillator strengths $\left(\mathrm{f}_{i j}\right.$, dimensionless), and line strengths ( $\mathrm{S}$, in atomic units) for electric dipole (E1), and $\mathrm{A}_{j i}$ for electric quadrupole (E2), magnetic dipole (M1), and magnetic quadrupole (M2) transitions of Ho LIX. The ratio R(E1) of velocity and length forms of $\mathrm{A}$-values for $\mathrm{E} 1$ transitions is listed in the last column.
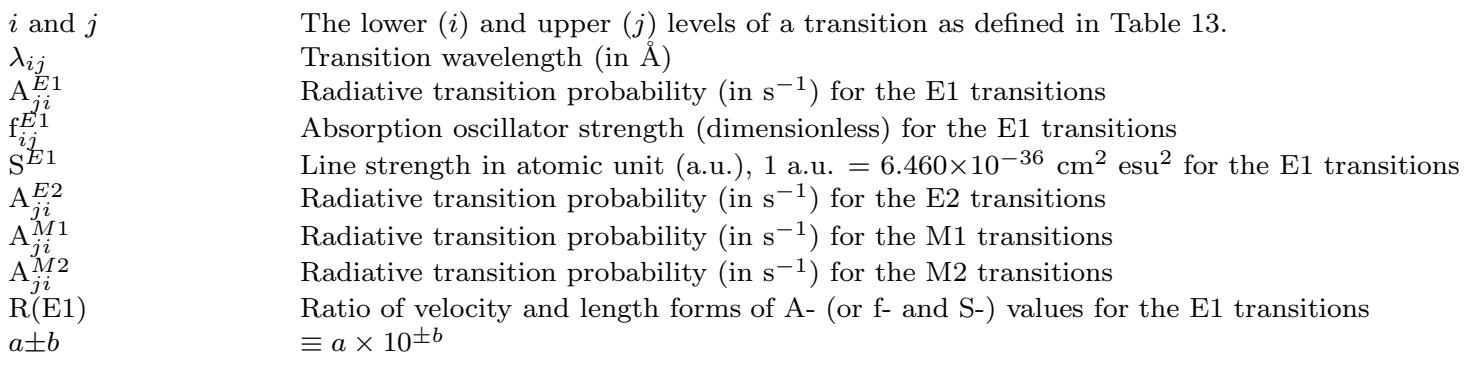

Table 33. Transition wavelengths $\left(\lambda_{i j}\right.$ in $\AA$ ), radiative rates $\left(\mathrm{A}_{j i}\right.$ in $\left.\mathrm{s}^{-1}\right)$, oscillator strengths $\left(\mathrm{f}_{i j}\right.$, dimensionless), and line strengths ( $\mathrm{S}$, in atomic units) for electric dipole (E1), and $\mathrm{A}_{j i}$ for electric quadrupole (E2), magnetic dipole (M1), and magnetic quadrupole (M2) transitions of Er LX. The ratio R(E1) of velocity and length forms of A-values for E1 transitions is listed in the last column.

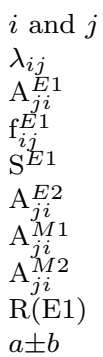

The lower $(i)$ and upper $(j)$ levels of a transition as defined in Table 14.

Transition wavelength (in $\AA$ )

Radiative transition probability (in $\mathrm{s}^{-1}$ ) for the E1 transitions

Absorption oscillator strength (dimensionless) for the E1 transitions

Line strength in atomic unit (a.u.), 1 a.u. $=6.460 \times 10^{-36} \mathrm{~cm}^{2} \mathrm{esu}^{2}$ for the E1 transitions

Radiative transition probability (in $\mathrm{s}^{-1}$ ) for the E2 transitions

Radiative transition probability (in $\mathrm{s}^{-1}$ ) for the M1 transitions

Radiative transition probability (in $\mathrm{s}^{-1}$ ) for the M2 transitions

Ratio of velocity and length forms of A- (or f- and S-) values for the E1 transitions

$\equiv a \times 10^{ \pm b}$ 
Table 34. Transition wavelengths $\left(\lambda_{i j}\right.$ in $\left.\AA\right)$, radiative rates $\left(\mathrm{A}_{j i}\right.$ in $\left.\mathrm{s}^{-1}\right)$, oscillator strengths $\left(\mathrm{f}_{i j}\right.$, dimensionless), and line strengths ( $\mathrm{S}$, in atomic units) for electric dipole (E1), and $\mathbf{A}_{j i}$ for electric quadrupole (E2), magnetic dipole (M1), and magnetic quadrupole (M2) transitions of Tm LXI. The ratio R(E1) of velocity and length forms of A-values for E1 transitions is listed in the last column.

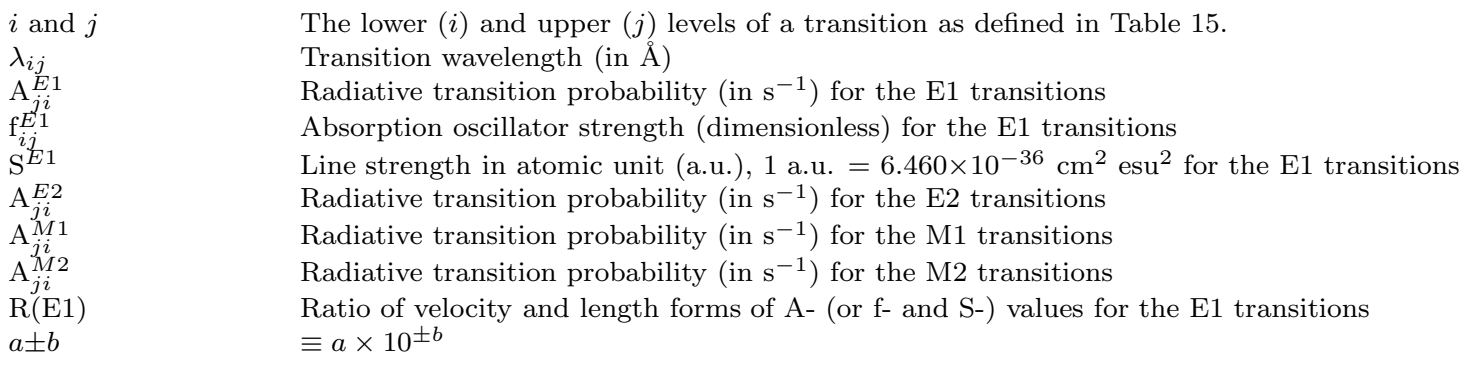

Table 35. Transition wavelengths $\left(\lambda_{i j}\right.$ in $\AA$ ), radiative rates $\left(\mathrm{A}_{j i}\right.$ in $\left.\mathrm{s}^{-1}\right)$, oscillator strengths $\left(\mathrm{f}_{i j}\right.$, dimensionless), and line strengths ( $\mathrm{S}$, in atomic units) for electric dipole (E1), and $\mathrm{A}_{j i}$ for electric quadrupole (E2), magnetic dipole (M1), and magnetic quadrupole (M2) transitions of Yb LXII. The ratio R(E1) of velocity and length forms of $\mathrm{A}$-values for $\mathrm{E} 1$ transitions is listed in the last column.
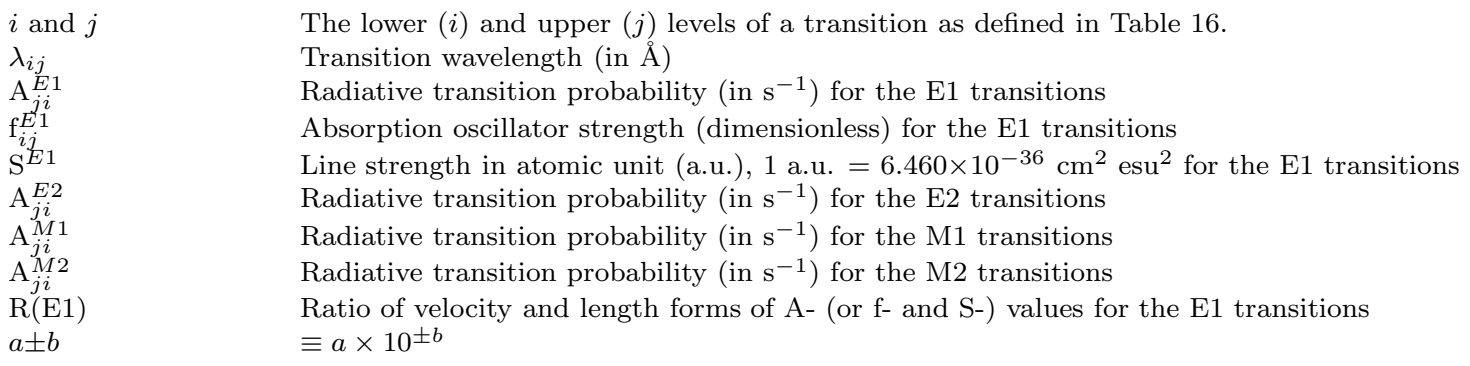

Table 36. Transition wavelengths $\left(\lambda_{i j}\right.$ in $\AA$ ) , radiative rates $\left(\mathrm{A}_{j i}\right.$ in $\left.\mathrm{s}^{-1}\right)$, oscillator strengths $\left(\mathrm{f}_{i j}\right.$, dimensionless), and line strengths ( $\mathrm{S}$, in atomic units) for electric dipole (E1), and $\mathrm{A}_{j i}$ for electric quadrupole (E2), magnetic dipole (M1), and magnetic quadrupole (M2) transitions of Lu LXIII. The ratio R(E1) of velocity and length forms of A-values for E1 transitions is listed in the last column.

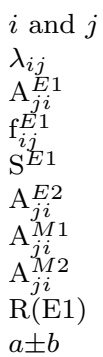

The lower $(i)$ and upper $(j)$ levels of a transition as defined in Table 17.

Transition wavelength (in $\AA$ )

Radiative transition probability (in $\mathrm{s}^{-1}$ ) for the E1 transitions

Absorption oscillator strength (dimensionless) for the E1 transitions

Line strength in atomic unit (a.u.), 1 a.u. $=6.460 \times 10^{-36} \mathrm{~cm}^{2} \mathrm{esu}^{2}$ for the E1 transitions

Radiative transition probability (in $\mathrm{s}^{-1}$ ) for the E2 transitions

Radiative transition probability (in $\mathrm{s}^{-1}$ ) for the M1 transitions

Radiative transition probability (in $\mathrm{s}^{-1}$ ) for the M2 transitions

Ratio of velocity and length forms of A- (or f- and S-) values for the E1 transitions

$\equiv a \times 10^{ \pm b}$ 
Table 37. Transition wavelengths $\left(\lambda_{i j}\right.$ in $\AA$ ), radiative rates $\left(\mathrm{A}_{j i}\right.$ in $\left.\mathrm{s}^{-1}\right)$, oscillator strengths $\left(\mathrm{f}_{i j}\right.$, dimensionless), and line strengths ( $\mathrm{S}$, in atomic units) for electric dipole (E1), and $\mathbf{A}_{j i}$ for electric quadrupole (E2), magnetic dipole (M1), and magnetic quadrupole (M2) transitions of Hf LXIV. The ratio R(E1) of velocity and length forms of A-values for E1 transitions is listed in the last column.

$i$ and $j$
$\lambda_{i j}$
$\mathrm{~A}_{j i}^{E 1}$
$\mathrm{f}_{i j}^{E 1}$
$\mathrm{~S}^{E 1}$
$\mathrm{~A}_{j i}^{E 2}$
$\mathrm{~A}_{j i}^{M 1}$
$\mathrm{~A}_{j i}^{M 2}$
$\mathrm{R}(\mathrm{E} 1)$
$a \pm b$

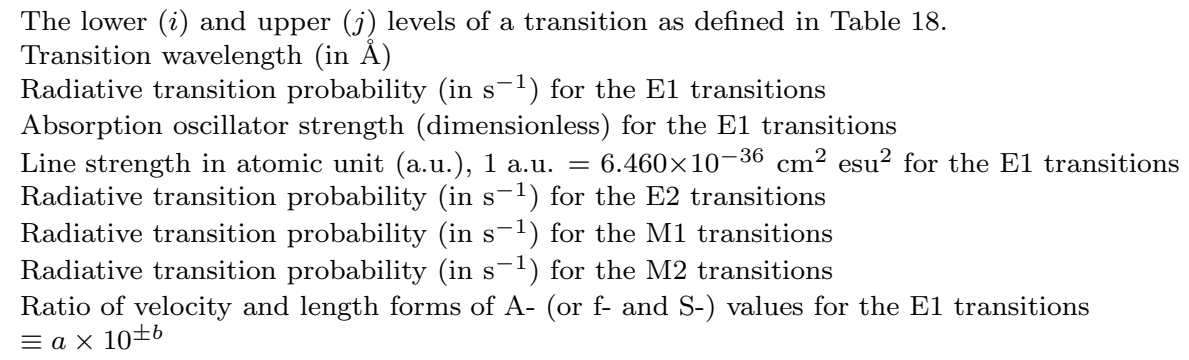

Table 38. Transition wavelengths $\left(\lambda_{i j}\right.$ in $\AA$ ), radiative rates $\left(\mathrm{A}_{j i}\right.$ in $\left.\mathrm{s}^{-1}\right)$, oscillator strengths $\left(\mathrm{f}_{i j}\right.$, dimensionless), and line strengths ( $\mathrm{S}$, in atomic units) for electric dipole (E1), and $\mathrm{A}_{j i}$ for electric quadrupole (E2), magnetic dipole (M1), and magnetic quadrupole (M2) transitions of Ta LXV. The ratio R(E1) of velocity and length forms of A-values for E1 transitions is listed in the last column. 